hep-th/0105208

\title{
Orientifolds with branes at angles
}

\author{
Stefan Förste, Gabriele Honecker, Ralph Schreyer \\ Physikalisches Institut, Universität Bonn \\ Nussallee 12, D-53115 Bonn, Germany
}

\begin{abstract}
We present supersymmetry breaking four dimensional orientifolds of type IIA strings. The compact space is a torus times a four dimensional orbifold. The orientifold group reflects one direction in each torus. RR tadpoles are cancelled by D6-branes intersecting at angles in the torus and in the orbifold. The angles are chosen such that supersymmetry is broken. The resulting four dimensional theories contain chiral fermions. The tadpole cancellation conditions imply that there are no non-abelian gauge anomalies. The models also contain anomaly-free $U(1)$ factors.
\end{abstract}




\section{Introduction}

One of the very important problems of string theory is the existence of a huge number of different vacua. As long as no dynamical mechanisms to select only a few (or even a unique) vacuum are known, one reasonable criterion to search for the correct vacuum is that in the low energy limit it should look similar to observations. This means that the resulting effective field theory should be the standard model or something "slightly beyond" the standard model. For a long time this criterion selected out especially Calabi-Yau compactifications of the ten dimensional heterotic string. These result in $N=1$ supersymmetric gauge theories coupled to gravity, which (as a category of theories) is fairly close to the standard model. After clarifying the importance of D-branes [1] and O-planes, the heterotic string lost its inimitability as a provider of phenomenologically interesting models. Now, gauge groups containing the standard model groups can appear due to open strings ending on D-branes. The appropriate tools to obtain lower dimensional (e.g. four dimensional) effective theories are orientifold constructions 2 [ 8 .

The strategy in constructing orientifold compactifications of type II strings is as follows. One starts with the compact space being an orbifold which is often taken to correspond to a limit in the space of Calabi-Yau manifolds. This breaks the amount of supersymmetry to twice the minimal amount in the low energy effective theory, and thus is not only "slightly" beyond the amount of supersymmetry in the standard model. Supersymmetry can be broken by another half when in addition worldsheet parity (possibly in combination with a discrete target space mapping) is gauged. Sets of points which are invariant under a group element containing the worldsheet parity inversion are called O-planes. These O-planes carry RR charges and typically possess only compact transverse directions. In order not to contradict RR charge conservation D-branes need to be added such that the net charge is zero. (This condition appears as the well known tadpole cancellation condition.) As long as the D-branes are parallel to the O-planes they do not break any further supersymmetry. Those orientifold compactifications give the minimal (apart from zero) amount of supersymmetry in the low energy effective action. Such models in various dimensions with and without chiral fermions, with and without D-branes at angles have been constructed. We give only a non comprehensive list of references here 925 .

In orientifold compactifications it may make sense to push the supersymmetry breaking one step further, to completely broken supersymmetry. The reason is that supersymmetry may not be necessary to explain the hierarchy between the Planck and the electro weak scale. In brane setups one can (sometimes) keep the fundamental scale (the string scale) at the weak scale and obtain the Planck scale by large compact extra dimensions 26.

There are essentially two ways to break supersymmetry completely by D-branes. Instead of satisfying the tadpole cancellation conditions by adding D-branes only one can also include anti-Dbranes (with opposite RR charges). Strings stretching between anti-D-branes and D-branes can have tachyonic excitations depending on the distance between the branes. Under certain circumstances the vacua are then unstable and undergo phase transitions 27 30]. Models of this kind have been presented e.g. in 3140 .

In this paper we will be interested in another way of supersymmetry breaking, namely via branes at angles. D-branes intersecting at angles generically break supersymmetry completely and only for very special values of the angles supersymmetry is partly unbroken. Such scenarios are e.g. discussed in 41 46]. We will mainly focus on the constructions in 41] and 44]. Indeed, we present basically a combination of these two models. Let us briefly describe the setup of [41] (we are interested in the four dimensional model they present). The compact space is a product of three tori $T^{2}$. The symmetry which is gauged is worldsheet parity inversion multiplied with the $\mathbb{Z}_{2}$ reflection of three compact dimensions, one in each torus. This is a symmetry of type IIA strings. Supersymmetry is completely broken by adding D6-branes which intersect at angles in each of the three tori. (The D6-branes have one Neumann and one Dirichlet direction in each torus.) From 
strings stretching between D-branes intersecting at angles in all the three tori chiral fermions are obtained. As argued in 41, 44 this setup has the disadvantage that an explanation of the hierarchy by having large extra dimensions is not possible in this model. The reason is that there is no "overall" transverse dimension which could be taken large. We will comment on this criticism below. But first we want to sketch the alternative model presented in [44]. Here, the compact space is chosen to be a $T^{2}$ times a four dimensional orbifold. The model contains D4-branes which are extended along the non-compact dimensions and in one direction in the $T^{2}$, where they intersect at angles. They are located at orbifold singularities in the remaining four compact directions. Again, chiral fermions arise from the sector where a string stretches between two D4-branes intersecting at angles. The hierarchy between the weak and the Planck scale can now be translated into a large orbifold volume.

In the present paper we combine these two constructions, i.e. we perform a further orbifold gauging of the model in 41, or (equivalently) an orientifold gauging of the model in 44]. The resulting low energy effective theories are non-supersymmetric and contain chiral fermions. However, like in the model of [41] there is no overall transverse dimension whose large volume could explain the hierarchy. But there may be a way out of this problem. The models are non-supersymmetric and free of RR tadpoles. This necessarily implies that they possess NSNS tadpoles, or roughly speaking that the net tension in the transverse space differs from zero. Taking into account the Fischler-Susskind mechanism 47 this non-vanishing net tension back-reacts on the excitations of the closed type IIA string, in particular on the metric. Such a back-reaction can result in a cosmological constant in the effective four dimensional theory but may also curve the geometry of the compact space. Stringy setups where this happens have been studied in 48, 49. For the kind of setups we discuss here the effect of the back-reaction has not been taken into account. This is perhaps a rather complicated computation. Qualitatively one would expect that the compact space curves. This affects the size of the four dimensional Planck mass, and the four dimensional gauge couplings differently. Such an effect may explain the hierarchy even if one starts without choosing some of the compact dimensions to be large, for a simple model where this happens see [50]. We are not claiming that this will happen in the models we discuss. However, it may be a way to obtain the scales in a natural way, about which we just miss sufficiently detailed knowledge.

The present paper is organized as follows. In the next section we line out the general setup. Section 3 provides the details of the construction. These are the possible torus lattices, the tadpole cancellation conditions and the massless spectra. In section 4 we discuss the differences due to the different orbifold projections and work out some examples explicitly. Section 5 contains a summary of our results and our conclusions. In two appendices we collect some formulæ which are used in the loop and tree channel computations of the tadpole cancellation conditions. A third appendix gives the massless closed string spectra and chiral fermions due to open strings in general and for a particular example.

\section{General Setup}

In this article we construct $\Omega \mathcal{R}$ orientifolds of type IIA string theory compactified to four dimensions on $T^{2} \times T^{4} / \mathbb{Z}_{N}$ with D6-branes at angles. In the following we explain some general features of these models and introduce some notation. For further details we refer to [23]. The four non-compact dimensions are labeled by $x^{\mu}, \mu=0, \ldots, 3$. The six compact directions we describe by three complex coordinates,

$$
z^{1}=x^{4}+i x^{5}, z^{2}=x^{6}+i x^{7}, z^{3}=x^{8}+i x^{9} .
$$


corresponding to three two-tori $T_{1,2,3}$. $\Omega$ reverses worldsheet parity, $\mathcal{R}$ reflects the imaginary parts of the $z^{i}$,

$$
\mathcal{R}: z^{i} \rightarrow \bar{z}^{i},
$$

and the $\mathbb{Z}_{N}$ rotation $\Theta$ acts as

$$
\Theta: z^{i} \rightarrow e^{2 \pi i v_{i}} z^{i}
$$

but on the tori $T_{2,3}$ only, i.e. $v_{1}=0$. The set of points invariant under $\Omega \mathcal{R} \Theta^{k}(k=0, \ldots, N-1)$ constitutes the orientifold fixed planes (O6-planes) which fill the non-compact dimensions and whose locations in the compact directions are shown in figure 1. The RR-charges of these O6-planes have

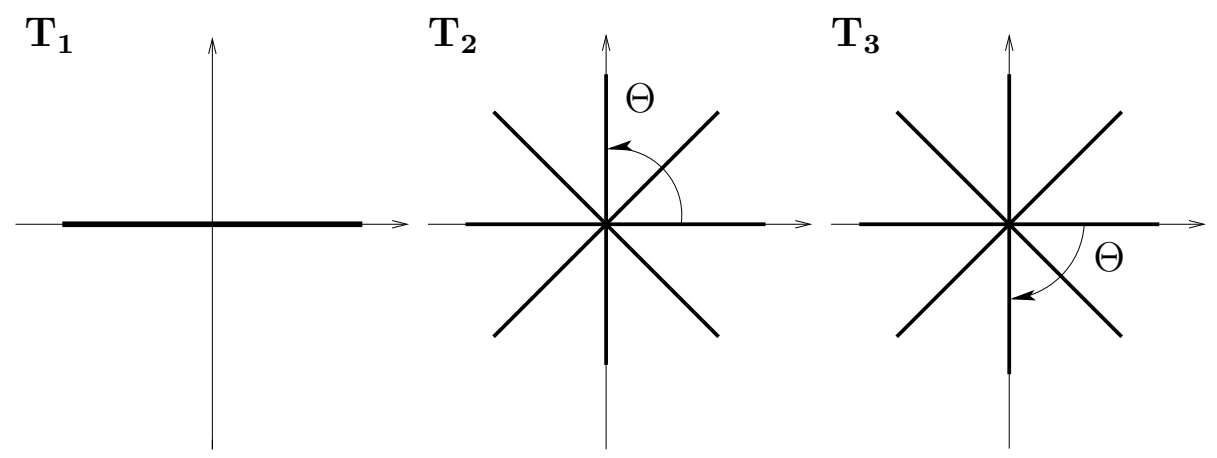

Figure 1: Orientifold fixed planes for $\mathbb{Z}_{4}$

to be cancelled by adding an appropriate arrangement of D6-branes. The most symmetric choice is to place the D6-branes on top of the O6-planes. This leads to a non-chiral and supersymmetric theory, in principle given by a simple torus compactification of the models discussed in 19. The requirement of balancing the RR-charges of O6-planes and D6-branes yields the tadpole cancellation conditions [8] which constitute the basic consistency conditions for orientifold constructions.

The tadpole cancellation conditions can be obtained in two different ways. One possibility is to extract the relevant UV-divergent parts of the loop channel diagrams which are given by the trace over the closed string NSNS states with $P \Omega \mathcal{R}(-)^{F}$ insertion, over the open string $\mathrm{R}$ states with $-P \Omega \mathcal{R}$ and over the open string NS states with $P(-)^{F}$ insertion in the case of the Klein bottle, the Möbius strip and the annulus, respectively. $P$ is the projector on the states invariant under the orbifold group $\mathbb{Z}_{N}$ and $(-)^{F}$ is the worldsheet fermion number. For further details again consider [23]. The second possibility is to construct the boundary states $|B\rangle$ for the D6-branes and the crosscap states $|C\rangle$ for the O6-planes [51] and to calculate directly the exchange of RR closed strings in the tree channel by the overlaps $\langle C|\ldots| C\rangle,\langle C|\ldots| B\rangle$ and $\langle B|\ldots| B\rangle$ in the case of the Klein bottle, the Möbius strip and the annulus, respectively. For further details, see appendix B.

The requirement that the amplitudes obtained in the two different ways explained above have to match after a modular transformation from the loop to the tree channel (or vice versa) is called worldsheet duality and further restricts the possible solutions. E.g. the Klein bottle amplitude restricts the possible choices of orbifold lattices, the Möbius strip yields conditions for the representation of the orientifold group on the Chan Paton matrices and from the annulus/cylinder one obtains the twisted sector tadpole cancellation conditions, since the identity $\Omega \mathcal{R} \Theta=\Theta^{-1} \Omega \mathcal{R}$ implies that only untwisted RR states may propagate in the tree channel. 


\section{Details of the construction}

Before presenting any calculations, we describe some general properties. The tori have to be invariant under the orientifold group, i.e. the torus $T_{1}$ has to be invariant under $\mathcal{R}$ whereas the tori $T_{2,3}$ have to be $\mathbb{Z}_{N}$ invariant in addition. The action of $\mathbb{Z}_{N}$ is defined via the shift $\vec{v}=(0,1 / N,-1 / N)$. The possible lattices are shown in the figures 2 and 3 for the example of $\mathbb{Z}_{4}$. In the case of $\mathbb{Z}_{2}$, the tori $T_{2,3}$ can be chosen to be of type $\mathbf{a}$ (or $\mathbf{b}$ ), too. We consider $K$ stacks of branes intersecting at

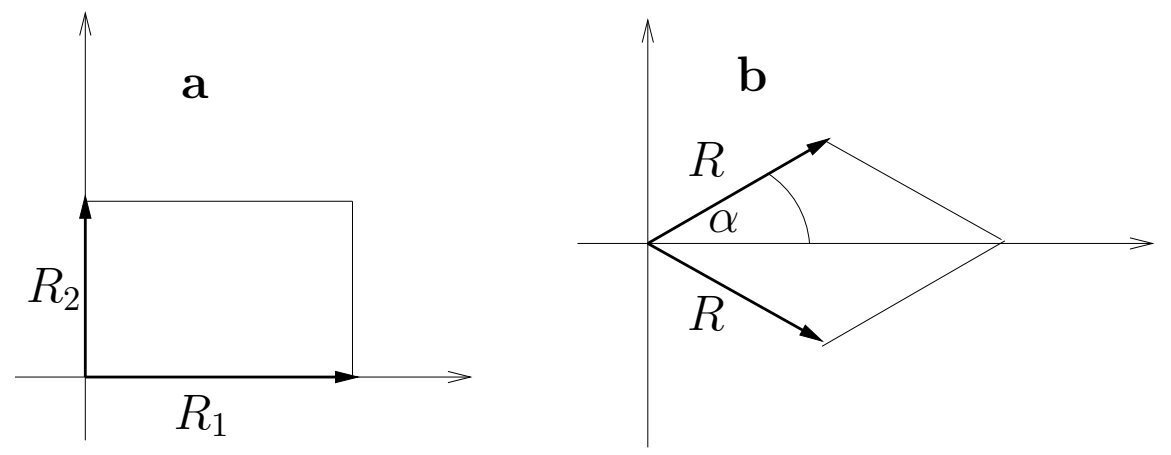

Figure 2: Possible torus lattices on $T_{1}$.
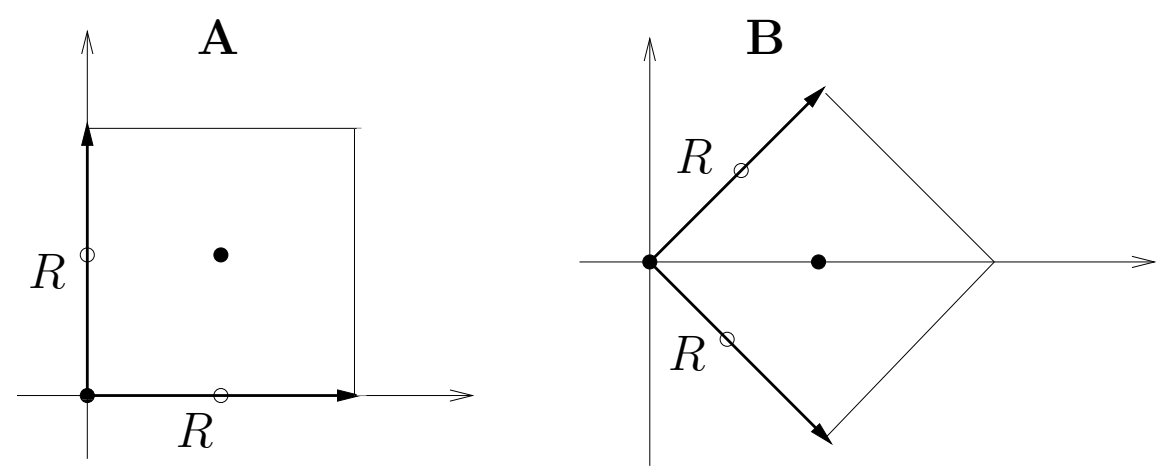

Figure 3: Possible torus lattices on $T_{2,3}$ for $\mathbb{Z}_{4}$. The black circles are the fixed points under $\Theta^{1,3}$ and the white circles are the additional fixed points under $\Theta^{2}$.

arbitrary angles on $T_{1}$. Each stack consists of $N_{a}(a=1, \ldots, K) \mathrm{D} 6_{a}$-branes on top of each other. In figure 4 an example is shown. On $T_{2,3}$ we choose the $\mathrm{D} 6_{a}$-branes to be arranged symmetrically (recall figure 1). The relative angles of the $\mathrm{D} 6_{a}$-branes with respect to the $x^{4}$ axis can be expressed in terms of the wrapping numbers $\left(n_{a}, m_{a}\right)$, i.e. the number of times the D6 $6_{a}$-branes wrap around the two fundamental cycles of $T_{1}$. We only consider models where the angles can be expressed in finite $\left(n_{a}, m_{a}\right)$, because otherwise the $\mathrm{D} 6_{a}$-brane would fill the $T_{1}$. Since $\mathcal{R}$ is a symmetry of the theory, the branes we have to consider actually are superpositions of $\mathrm{D} 6_{a}$-branes with their mirror branes with respect to the $x^{4}$ axis. Therefore we may choose $m_{a} \geq 0$. Furthermore, the choice $n_{a}>0$ reflects the fact that we do not consider $\overline{\mathrm{D}}_{a}$-branes in this paper. As one can see in figure $\mathbb{\theta}$, in general the branes intersect plurally on $T_{1}$. The number of intersections of two stacks of branes D6 $6_{a}$ and $\mathrm{D}_{b}$ can be expressed in terms of the wrapping numbers

$$
I_{a b}=n_{a} m_{b}-m_{a} n_{b},
$$




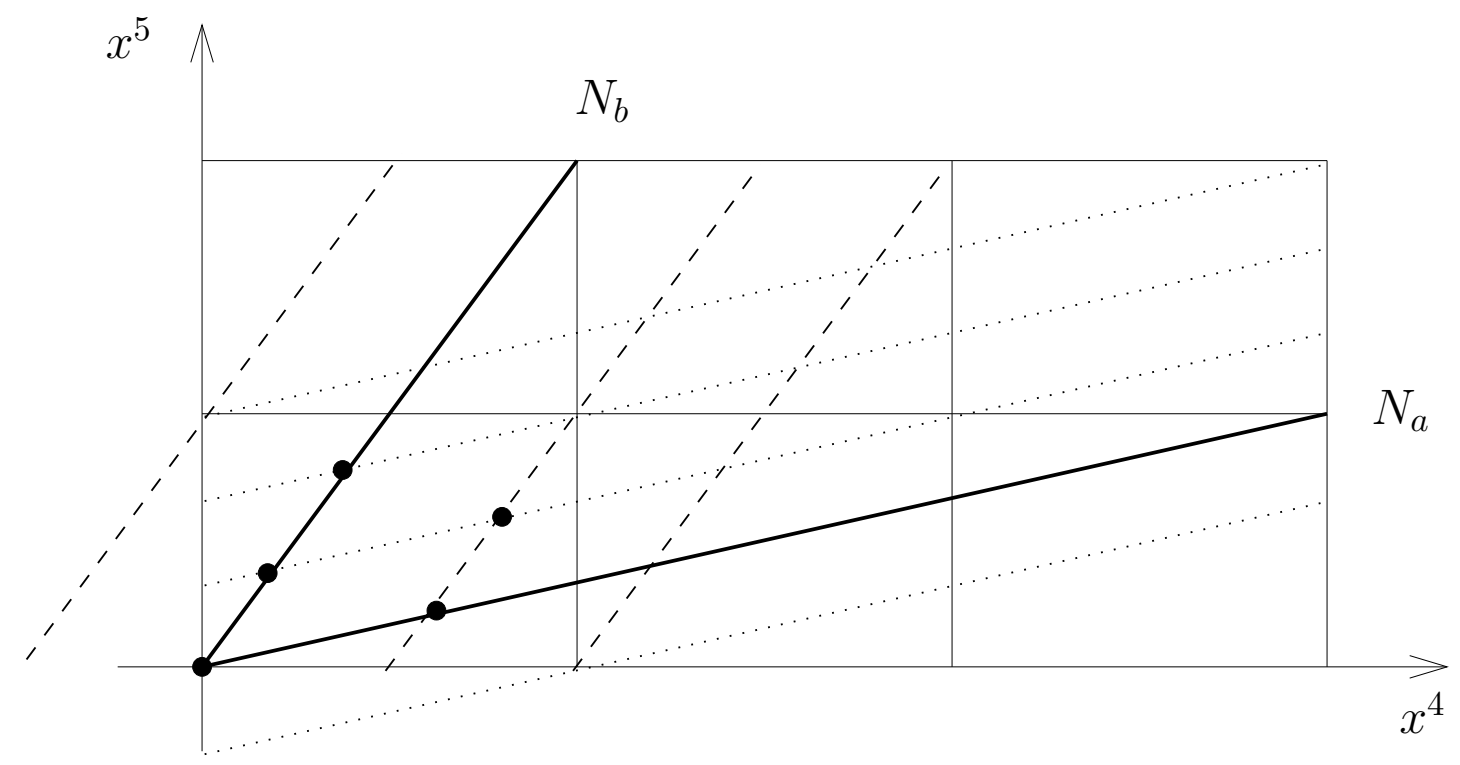

Figure 4: One stack of $N_{a} \mathrm{D} 6_{a}$-branes with wrapping number $\left(n_{a}, m_{a}\right)=(3,1)$ intersecting another stack of $N_{b} \mathrm{D} 6_{b}$-branes with wrapping number $\left(n_{b}, m_{b}\right)=(1,2)$ on $T_{1}$. The black circles denote the multiple intersection points on the torus.

as one can check for the example in figure A. A negative wrapping number makes no sense geometrically, but one can get rid of the negative sign by choosing the conjugate representation for the corresponding particles.

\subsection{Amplitudes and tadpole cancellation}

We start with the Klein bottle loop channel amplitude. The compact momenta consist of Kaluza Klein contributions

$$
P=r_{1} \vec{e}_{1}^{*}+r_{2} \vec{e}_{2}^{*}
$$

and contributions from windings

$$
\alpha^{\prime} W=s_{1} \vec{e}_{1}+s_{2} \vec{e}_{2},
$$

where the $r_{i}, s_{i}$ are integers and the $\vec{e}_{i}\left(\vec{e}_{i}^{*}\right)$ are the basis vectors of the (dual) torus lattice. Requiring invariance of $P$ and $W$ under the orientifold group and using $\Omega P \Omega^{-1}=P$ and $\Omega W \Omega^{-1}=$ $-W$ one obtains the lattice contributions to the amplitude $\mathcal{L}^{R_{1}, R_{2}}[1,1]$ for the a type $T_{1}$ and $\mathcal{L}^{R, R}\left[1 / \cos ^{2} \alpha, 4 \sin ^{2} \alpha\right]$ for the $\mathbf{b}$ type $T_{1}$. On $T_{2,3}$ one has to set $R_{1}=R_{2}=R$ for $\mathbf{A}$ and $\alpha=\pi / 4$ for $\mathbf{B}$ in the case of $\mathbb{Z}_{4}$, for example (see appendix A.1 for the notation). Of course, in general the radii of the different tori are not the same, so there should be further indices for the radii, but we omit them to keep the notation simple. The different lattice contributions are summarized in table 4 . Lattice contributions only show up in the untwisted sector. One could think that all possible combinations aAA, bAA etc. are allowed, but here worldsheet duality comes into the game, see [19, 23] and appendix $\mathbb{B}$. In the case of $\mathbb{Z}_{4}\left(\right.$ and $\mathbb{Z}_{6}$ ), an insertion of $\Theta$ in the trace interchanges contributions from A and B. But since, as stated above, in the tree channel only untwisted RR states propagate, each contribution in the loop channel has to be invariant under interchange of $\mathbf{A}$ and $\mathbf{B}$. Thus in the cases of $\mathbb{Z}_{4,6}$ only the models aAB and $\mathbf{b A B}$ (which are equivalent to aBA and bBA, respectively), whereas for $\mathbb{Z}_{2,3}$ all combinations are allowed. 
The calculation of the NSNS part with $(-)^{F}$ insertion of the one loop amplitude yields

$$
\mathcal{K}=c \int_{0}^{\infty} \frac{d t}{t^{3}} \mathcal{L}_{1}^{\mathcal{K}}\left(\mathcal{K}^{(0)} \mathcal{L}_{2}^{\mathcal{K}} \mathcal{L}_{3}^{\mathcal{K}}+\sum_{n=1}^{N-1} \chi^{(n)} \mathcal{K}^{(n)}\right)
$$

where $\mathcal{K}^{(n)}$ are the oscillator contributions from the $n$th twisted sector (see [21, 23] for the notation), $\chi^{(n)}$ are the multiplicities from the orbifold fixed points and $c=V_{4} /\left(8 \pi^{2} \alpha^{\prime}\right)^{2}$ is the regularized volume factor from the non-compact momentum integration. Note that the result (7) already implies the consequences from worldsheet duality, namely the fact that not all combinations of lattices are allowed, as stated above.

Performing the modular transformation using $t=1 / 4 l$ leads to the tree channel amplitude

$$
\tilde{\mathcal{K}}=c c_{1}^{\mathcal{K}} c_{2}^{\mathcal{K}} c_{3}^{\mathcal{K}} \int_{0}^{\infty} d l \tilde{\mathcal{L}}_{1}^{\mathcal{K}} \frac{\vartheta\left[\begin{array}{c}
1 / 2 \\
0
\end{array}\right]^{2}}{\eta^{6}}\left\{\tilde{\mathcal{L}}_{2}^{\mathcal{K}} \tilde{\mathcal{L}}_{3}^{\mathcal{K}} \frac{\vartheta\left[\begin{array}{c}
1 / 2 \\
0
\end{array}\right]^{2}}{\eta^{6}}-4 \sum_{n=1}^{N-1} \sin ^{2}\left(\frac{\pi n}{N}\right) \frac{\vartheta\left[\begin{array}{c}
1 / 2 \\
n / N
\end{array}\right] \vartheta\left[\begin{array}{c}
1 / 2 \\
-n / N
\end{array}\right]}{\vartheta\left[\begin{array}{c}
1 / 2 \\
1 / 2+n / N
\end{array}\right] \vartheta\left[\begin{array}{c}
1 / 2 \\
1 / 2-n / N
\end{array}\right]}\right\}
$$

corresponding to the $\mathrm{RR}$ exchange in the tree channel. The $c_{i}^{\mathcal{K}}$ can be read off from $\mathcal{L}_{i}^{\mathcal{K}}=c_{i}^{\mathcal{K}} l \tilde{\mathcal{L}}_{i}^{\mathcal{K}}$ and are displayed in table $\mathbb{6}$. The argument of the $\vartheta$ and $\eta$ functions in equation (\&) is given by $e^{-4 \pi l}$.

Calculating the amplitude directly in the tree channel using the boundary state formalism [51] and comparing the result with equation (8) (i.e. requiring worldsheet duality) restricts the possible choice of the tori, as stated above, and determines the normalization of the crosscap states to be

$$
\mathcal{N}_{C}=\sqrt{\frac{c c_{1}^{\mathcal{K}} c_{2}^{\mathcal{K}} c_{3}^{\mathcal{K}}}{2 N}}
$$

see appendix B.1 for the details and notation.

Now we consider the annulus amplitude, i.e. the one-loop amplitude from strings starting and ending on some of the D6-branes we have to add to cancel the tadpoles arising from the orientifold planes. Remember that we add $N_{a} \mathrm{D} 6_{a}$-branes $(a=1, \ldots, K)$ at some angles on $T_{1}, \mathbb{Z}_{N}$ symmetric on $T_{2,3}$. Let us first discuss the one-loop amplitude from strings starting and ending on the same stack of $\mathrm{D} 6_{a}$-branes. The compact momenta corresponding to the Kaluza Klein momenta in the case of the Klein bottle are given by the inverse length of the D6-branes on the corresponding torus. Thus, e.g. for the a type $T_{1}$ one obtains

$$
P=\frac{r}{\sqrt{\left(n_{a} R_{1}\right)^{2}+\left(m_{a} R_{2}\right)^{2}}} \equiv r V^{-1}
$$

where $r$ is an integer. Strictly speaking, $P$ and $V$ should carry the index of the wrapping numbers, but we omit this index here and in the following. The compact momenta corresponding to the windings in the case of the Klein bottle are given by the distance of parallel D6-branes on the corresponding torus, see figure 4 . For the a type $T_{1}$ one obtains

$$
\alpha^{\prime} W=s R_{1} R_{2} V^{-1}
$$

with $s$ integer. The lattice contributions then appear as $\mathcal{L}_{\mathbf{a}}^{\mathcal{A}}=\sum_{r, s} e^{-2 \pi t \alpha^{\prime} M^{2}}$ with $M^{2}=P^{2}+$ $\left(\alpha^{\prime} W\right)^{2}$ in the trace. The results for the $\mathbf{b}$ type $T_{1}$ are shown in table 4 . The relevant part of the one loop amplitude from $\mathrm{D} 6_{a}-\mathrm{D} 6_{a}$ strings reads

$$
\mathcal{A}_{a a}=\frac{c}{4} N_{a}^{2} \int_{0}^{\infty} \frac{d t}{t^{3}} \mathcal{L}_{1}^{\mathcal{A}} \frac{\vartheta\left[\begin{array}{c}
0 \\
1 / 2
\end{array}\right]^{2}}{\eta^{6}}\left\{\mathcal{L}_{2}^{\mathcal{A}} \mathcal{L}_{3}^{\mathcal{A}} \frac{\vartheta\left[\begin{array}{c}
0 \\
1 / 2
\end{array}\right]^{2}}{\eta^{6}}+\sum_{n=1}^{N-1} \chi_{\mathcal{A}}^{(n)} \frac{\vartheta\left[\begin{array}{c}
n / N \\
1 / 2
\end{array}\right] \vartheta\left[\begin{array}{c}
-n / N \\
1 / 2
\end{array}\right]}{\vartheta\left[\begin{array}{c}
1 / 2+n / N \\
1 / 2
\end{array}\right] \vartheta\left[\begin{array}{c}
1 / 2-n / N \\
1 / 2
\end{array}\right]}\right\}
$$


where $\chi_{\mathcal{A}}^{(n)}$ is the intersection number of the D6-branes on $T_{2,3}$. The argument of the $\vartheta$ and $\eta$ functions is $e^{-\pi t}$. Equation (12) already contains another consequence of worldsheet duality, namely the fact that the Chan Paton representations of $\mathbb{Z}_{2}$ elements of the orbifold group have to be traceless. This is the so called "twisted tadpole cancellation condition".

The modular transformation $t=1 / 2 l$ leads to

$$
\tilde{\mathcal{A}}_{a a}=\frac{c}{2^{4}} N_{a}^{2} c_{1}^{\mathcal{A}} c_{2}^{\mathcal{A}} c_{3}^{\mathcal{A}} \int_{0}^{\infty} d l \tilde{\mathcal{L}}_{1}^{\mathcal{A}} \frac{\vartheta\left[\begin{array}{c}
1 / 2 \\
0
\end{array}\right]^{2}}{\eta^{6}}\left\{\tilde{\mathcal{L}}_{2}^{\mathcal{A}} \tilde{\mathcal{L}}_{3}^{\mathcal{A}} \frac{\vartheta\left[\begin{array}{c}
1 / 2 \\
0
\end{array}\right]^{2}}{\eta^{6}}-4 \sum_{n=1}^{N-1} \cdots\right\}
$$

where $-4 \sum_{n=1}^{N-1} \ldots$ is just the same as in equation (8). The argument of the $\vartheta$ and $\eta$ functions is $e^{-4 \pi l}$. Comparing this with the result from the boundary state formalism, one obtains the normalization factor for the boundary states

$$
\mathcal{N}_{B}=\sqrt{\frac{c c_{1}^{\mathcal{A}} c_{2}^{\mathcal{A}} c_{3}^{\mathcal{A}}}{2^{5} N}}
$$

see appendix B.2 for the details and notation.

Having found the complete boundary and crosscap states, the calculation of the remaining amplitudes becomes a straightforward task. The tree level cylinder amplitude from strings stretched between the branes $\mathrm{D} 6_{a}$ and $\mathrm{D} 6_{b}$ intersecting at a relative angle $\pi \Delta \varphi$ reads

$$
\tilde{\mathcal{A}}_{a b}=\frac{c}{2^{3}} N_{a} N_{b} I_{a b} c_{2}^{\mathcal{A}} c_{3}^{\mathcal{A}} \int_{0}^{\infty} d l \frac{\vartheta\left[\begin{array}{c}
1 / 2 \\
0
\end{array}\right]}{\eta^{3}} \frac{\vartheta\left[\begin{array}{c}
1 / 2 \\
\Delta \varphi
\end{array}\right]}{\vartheta\left[\begin{array}{c}
1 / 2 \\
1 / 2+\Delta \varphi
\end{array}\right]}\left\{\tilde{\mathcal{L}}_{2}^{\mathcal{A}} \tilde{\mathcal{L}}_{3}^{\mathcal{A}} \frac{\vartheta\left[\begin{array}{c}
1 / 2 \\
0
\end{array}\right]^{2}}{\eta^{6}}-4 \sum_{n=1}^{N-1} \ldots\right\}
$$

where $I_{a b}$ is the intersection number of $\mathrm{D} 6_{a}$ and $\mathrm{D} 6_{b}$ on $T_{1}$ defined in equation (4).

The contributions from the Möbius strip to the RR exchange in the tree channel are obtained by calculating the overlap of the corresponding boundary and crosscap states. We first discuss the case of a string starting on a D6-brane aligned with the $x^{4}$-axis on $T_{1}$ and ending on the corresponding O6-plane (and vice versa). The relevant part of the amplitude is given by

$$
\tilde{\mathcal{M}}_{\|}=-\frac{c}{2^{4}} N_{a} c_{1}^{\mathcal{M}} c_{2}^{\mathcal{M}} c_{3}^{\mathcal{M}} \int_{0}^{\infty} d l \tilde{\mathcal{L}}_{1}^{\mathcal{M}} \frac{\vartheta\left[\begin{array}{c}
1 / 2 \\
0
\end{array}\right]^{2}}{\eta^{6}}\left\{\tilde{\mathcal{L}}_{2}^{\mathcal{M}} \tilde{\mathcal{L}}_{3}^{\mathcal{M}} \frac{\vartheta\left[\begin{array}{c}
1 / 2 \\
0
\end{array}\right]^{2}}{\eta^{6}}-4 \sum_{n=1}^{N-1} \ldots\right\} .
$$

The argument of the $\vartheta$ and $\eta$ functions is $-e^{-4 \pi l}$. Similarly, we obtain the relevant contribution from a string starting (or ending) on a $\mathrm{D} 6_{a}$-brane at angle $\pi \varphi$ with respect to the $x^{4}$-axis on $T_{1}$

$$
\tilde{\mathcal{M}}_{a}=-\frac{c}{2^{2}} N_{a} I_{a^{\prime} a}^{\Omega \mathcal{R}} c_{2}^{\mathcal{M}} c_{3}^{\mathcal{M}} \int_{0}^{\infty} d l \frac{\vartheta\left[\begin{array}{c}
1 / 2 \\
0
\end{array}\right]}{\eta^{3}} \frac{\vartheta\left[\begin{array}{c}
1 / 2 \\
\varphi
\end{array}\right]}{\vartheta\left[\begin{array}{c}
1 / 2 \\
1 / 2+\varphi
\end{array}\right]}\left\{\tilde{\mathcal{L}}_{2}^{\mathcal{M}} \tilde{\mathcal{L}}_{3}^{\mathcal{M}} \frac{\vartheta\left[\begin{array}{c}
1 / 2 \\
0
\end{array}\right]^{2}}{\eta^{6}}-4 \sum_{n=1}^{N-1} \ldots\right\}
$$

where $I_{a^{\prime} a}^{\Omega \mathcal{R}}$ is the number of $\Omega \mathcal{R}$-invariant intersections of the brane $\mathrm{D} 6_{a}$ with its $\Omega \mathcal{R}$-image $\mathrm{D} 6_{a^{\prime}}$.

Putting everything together and taking the limit $l \rightarrow \infty$, we obtain the tadpole cancellation 
conditions

$$
\begin{aligned}
& \mathbb{Z}_{2}: \quad \sum_{a} N_{a} n_{a}=\left\{\begin{array}{cc}
16 & (\mathbf{a a a}) \\
8 & (\mathbf{a a b}) \\
4 & (\mathbf{a b b})
\end{array}\right. \\
& \mathbb{Z}_{3}: \quad \sum_{a} N_{a} n_{a}=4 \quad(\mathbf{a A A}, \mathbf{a A B}, \mathbf{a B B}) \\
& \mathbb{Z}_{4}: \quad \sum_{a} N_{a} n_{a}=8 \quad(\mathbf{a A B}) \\
& \mathbb{Z}_{6}: \quad \sum_{a} N_{a} n_{a}=4 \quad(\mathbf{a A B})
\end{aligned}
$$

for the $\mathbf{b}$ type $T_{1}$ one just has to replace $n_{a}$ by $n_{a}+m_{a}$. Since we are restricted to the cases $n_{a}>0$ and $m_{a} \geq 0$, there is not much freedom left for the construction of models. In section 1 we give some explicit examples.

\subsection{The spectrum}

Generically, we obtain non-supersymmetric and chiral models which contain tachyons. The only exception is the trivial case, where $m_{a}=0$ for all $a$, where the spectrum is $\mathcal{N}=2$ supersymmetric, nonchiral and free of tachyons.

In the untwisted closed string sector we find the $\mathcal{N}=2$ SUGRA multiplet. The twisted closed string sectors and the sectors from open strings which do not stretch between D6-branes at angles on $T_{1}$ contain further $\mathcal{N}=2 \mathrm{SUSY}$ multiplets. What makes the spectrum generically nonsupersymmetric, are the open strings stretching between branes at angles on $T_{1}$. In these sectors we always find tachyons. We find chiral fermions not only in the sectors where the strings stretch between branes at angles on all three tori, but also where they stretch between branes at angles on $T_{1}$ only, if an additional $\mathbb{Z}_{2}$ symmetry from the orbifold group is present, because in this case the left- and right-handed states are distinguished by different parity under the $\mathbb{Z}_{2}$ transformation. The appearence of tachyons can easily be seen considering the mass formula for open strings in the NS sector

$$
\alpha^{\prime} m^{2}=\operatorname{osc}+\frac{1}{2}\left(\Delta \varphi+2 \frac{k}{N}-1\right)
$$

where $-1 / 2<\Delta \varphi<1 / 2$ is the relative angle on $T_{1}$ and $k / N$ is the relative angle on $T_{2,3}$ in units

of $\pi$. "osc" is minus the moding of the applied oscillator, thus e.g. for the state $\psi_{\Delta \varphi-1 / 2}^{1}|0\rangle$ from a string with $k=0$ we obtain a tachyon with $\alpha^{\prime} m^{2}=-\Delta \varphi / 2$. The resulting gauge groups are

$$
\begin{array}{ll}
\mathbb{Z}_{2,4,6}: & \prod_{m_{a} \neq 0} U\left(N_{a} / 2\right)^{4} \prod_{m_{a}=0} U\left(N_{a} / 2\right)^{2} \\
\mathbb{Z}_{3}: & \prod_{m_{a} \neq 0} U\left(N_{a}\right) \prod_{m_{a}=0} S O\left(N_{a}\right),
\end{array}
$$

for further details see appendix $\mathrm{C}$ and the explicit examples in the following section.

\section{$4 \quad$ The $\mathbb{Z}_{3}$ and $\mathbb{Z}_{2}$ case}

\subsection{Models on $T^{2} \times T^{4} / \mathbb{Z}_{3}$}

The most simple model on $T^{2} \times T^{4} / \mathbb{Z}_{N}$ is the case $N=3$ where the branes lie on top of the O-planes on $T_{2,3}$ (similar to figure 11). In the case of $\mathbb{Z}_{3}, \Theta^{1 / 2}$ is a symmetry of the lattice and therefore all 
branes wrapping the same cycle on $T_{1}$ are identified under the orbifold group [23, 19]. Generically, a set of branes with $\left(n_{a}, m_{a}\right)$ generates a $U\left(N_{a}\right)$ gauge group whereas for $\left(n_{a}, m_{a}\right)=(1,0)$, the branes have an additional $\Omega \mathcal{R}$-symmetry. Imposing the corresponding projection condition breaks the gauge group down to $S O\left(N_{a}\right)$.

We can choose the lattice orientations $\mathbf{A}, \mathbf{B}$ independently on $T_{2,3}$. The tadpole cancellation condition (18) is not affected by this choice, but the chiral fermionic spectrum listed in table 1 receives an overall multiplicity $\chi$ from the number of brane intersections in the fundamental cell. As $\Theta$ and $\Theta^{2}$ sectors yield the same contribution, each fermion comes in an even number of copies.

Strings starting and ending on stacks of branes $a, b$ with different wrappings on $T_{1}$ transform in the bifundamental of $U\left(N_{a}\right) \times U\left(N_{b}\right)$. $\Omega \mathcal{R}$ simply maps $a b$-strings to $b^{\prime} a^{\prime}$-strings. In the special case of strings stretching between mirror branes $a a^{\prime}$, we have to analyse the intersection points more closely. On $T_{2,3}$, all intersections are invariant whereas on $T_{1}$ there are $2 m_{a}$ points which are mapped onto themselves by the reflection $\mathcal{R}$ while the remaining $2 m_{a}\left(n_{a}-1\right)$ form pairs. The latter support fermions in the antisymmetric $\left(\mathbf{A}_{a}\right)$ and symmetric $\left(\mathbf{S}_{a}\right)$ representation. At $\Omega \mathcal{R}$ invariant brane intersections, the symmetric part is projected out and we only obtain fermions transforming in the antisymmetric representation. The generic chiral fermionic spectrum as displayed in table 1 is free of purely non-abelian gauge anomalies. This is a consequence of the tadpole cancellation condition (18).

\begin{tabular}{|c||c|c|}
\hline \multicolumn{2}{|c|}{ Chiral fermionic spectrum for $\mathbb{Z}_{3}$} \\
\hline \hline & rep. & mult. \\
\hline \hline$a a^{\prime}$ & $\left(\mathbf{A}_{a}\right)_{L}$ & $4 m_{a} \chi$ \\
$a a^{\prime}$ & $\left(\mathbf{A}_{a}\right)_{L}+\left(\mathbf{S}_{a}\right)_{L}$ & $2 m_{a}\left(n_{a}-1\right) \chi$ \\
$a b$ & $\left(\overline{\mathbf{F}}_{a}, \mathbf{F}_{b}\right)_{L}$ & $2\left(n_{a} m_{b}-n_{b} m_{a}\right) \chi$ \\
$a b^{\prime}$ & $\left(\mathbf{F}_{a}, \mathbf{F}_{b}\right)_{L}$ & $2\left(n_{a} m_{b}+n_{b} m_{a}\right) \chi$ \\
\hline
\end{tabular}

Table 1: Generic chiral spectrum for $\mathbb{Z}_{3} \cdot \quad \chi=1(3,9)$ is the intersection number on $T_{2,3}$ for the lattices AA (AB, BB).

\subsubsection{Example 1, $\mathbb{Z}_{3}$}

As we restrict our analysis to $\mathrm{D} 6_{a}$-branes, the largest feasible gauge group which respects the tadpole condition (18) and yields chiral fermions is $U(3) \times U(1)$. We can split this group into $S U(3) \times U(1)^{2}$. Choosing the wrapping numbers $\left(n_{1}, m_{1}\right)=(1,1),\left(n_{2}, m_{2}\right)=(1,2)$, out of the two $U(1)$ 's the linear combination

$$
Q_{\text {non-an. }}=Q_{1}-\frac{3}{2} Q_{2}
$$

is non-anomalous. The remaining $U(1)$ should get a mass of the order of the string scale by a generalized Green-Schwarz mechanism involving closed string moduli [44, 52] (and references therein). The resulting spectrum is displayed in table 2 for the lattice aAA.

\subsection{Models on $T^{2} \times T^{4} / \mathbb{Z}_{2}$}

$\mathbb{Z}_{2}$ models are the most simple examples for $T^{4} / \mathbb{Z}_{M}$ orbifolds where $M$ is even. In this case, the requirement of obtaining the complete projector in the tree channel leads to two tadpole cancellation 


\begin{tabular}{|c||c|c|}
\hline \multicolumn{3}{|c|}{ Chiral spectrum, Ex. 1} \\
\hline \hline & $S U(3) \times U(1)_{\text {non-an. }}$ & mult. \\
\hline \hline $11^{\prime}$ & $(\overline{\mathbf{3}})_{2}$ & 4 \\
12 & $(\overline{\mathbf{3}})_{-5 / 2}$ & 2 \\
$12^{\prime}$ & $(\mathbf{3})_{-1 / 2}$ & 6 \\
\hline
\end{tabular}

Table 2: Chiral fermionic spectrum for $\mathbb{Z}_{3}$ with $\left(n_{1}, m_{1}\right)=(1,1),\left(n_{2}, m_{2}\right)=(1,2)$ and lattice aAA. All states are left-handed.

conditions, namely

$$
\begin{aligned}
\sum_{a} \chi n_{a} N_{a} & =16 \\
\operatorname{tr} \gamma_{\Theta} & =0
\end{aligned}
$$

where $\chi=1(2,4)$ is the intersection number on $T_{2,3}$ for $\mathbf{a a}(\mathbf{a b}, \mathbf{b} \mathbf{b})$.

There are three major differences as compared to section 4.1: First of all, worldsheet duality gives a constraint on the representation matrices of the orbifold group leading to a smaller gauge group, $U\left(N_{a}\right) \stackrel{\mathbb{Z}_{2}}{\longrightarrow} U\left(N_{a} / 2\right) \times U\left(N_{a} / 2\right)\left[\stackrel{\Omega \mathcal{R}}{\longrightarrow} U\left(N_{a} / 2\right)\right.$ for a brane which is its own mirror brane]. Secondly, for a given wrapping number on $T_{1}$ there exist two different brane configurations on $T_{2,3}$, namely those extended along the $\left(x^{6}, x^{8}\right)$ - and those along the $\left(x^{7}, x^{9}\right)$-directions, which cannot be identified by any element of the orientifold group. Thus, for given $\left(n_{a}, m_{a}\right)$ we obtain the total gauge group $U\left(N_{a} / 2\right)^{4}\left[U\left(N_{a} / 2\right)^{2}\right.$ for $\left.m_{a}=0\right]$. At last, the intersection number $\chi$ on $T_{2,3}$ explicitly enters the tadpole cancellation condition. $\quad \mathrm{A} \mathbb{Z}_{2}$ rotation maps each sector onto itself whilst assigning a fixed parity \pm 1 to each massless state. As one can easily read off from table 5, left-handed states are $\mathbb{Z}_{2}$-even and right-handed ones are $\mathbb{Z}_{2}$-odd in all sectors with a non-vanishing angle on $T_{1}$. Therefore, not only strings stretching between branes at non-vanishing angles on all three tori contribute to the chiral spectrum but also those which are merely tilted on $T_{1}$. This is also the reason why the intersection number $\chi$ explicitly enters the tadpole cancellation conditions (22). Taking all this into account, the chiral part of the open string spectrum is as listed in table 3 .

The analysis of the $\mathbb{Z}_{4,6}$ cases goes completely analogous to $\mathbb{Z}_{2}$. Again, there exist two independent brane configurations on $T_{2,3}$ and a condition on the Chan-Paton matrices, $\operatorname{tr} \gamma_{\Theta^{N / 2}}=0$, yielding the gauge group $U\left(N_{a} / 2\right)^{4}$ for given $\left(n_{a}, m_{a}\right)$. "Untwisted" strings on $T_{2,3}$ will contribute to the chiral spectrum since $\mathbb{Z}_{2}$-even and -odd states have opposite handedness. One additional subtlety enters the computation of the " $\Theta^{2}$-twisted" and " $\Theta^{3}$-twisted" open spectrum in the case of $\mathbb{Z}_{6}$ as the intersection points on $T_{2,3}$ are permuted by the orbifold group. However, the tadpole conditions (18) already indicate that we cannot include the standard model gauge group $S U(3) \times S U(2) \times U(1)$ in $\mathbb{Z}_{4,6}$ without adding anti-branes. Therefore, we will not discuss these models in detail but close this section by giving a $\mathbb{Z}_{2}$ example which encloses $S U(3) \times S U(2) \times U(1)$. 


\begin{tabular}{|c|c|c|}
\hline \multicolumn{3}{|c|}{ Chiral fermionic spectrum for $\mathbb{Z}_{2}$} \\
\hline & rep. of $U\left(\frac{N_{a}}{2}\right)^{4} \times U\left(\frac{N_{b}}{2}\right)^{4}$ & mult. \\
\hline$a a^{\prime} U$ & $\begin{array}{c}\left(\mathbf{F}_{a}, \mathbf{F}_{a}, 1,1\right)_{L}+\left(1,1, \mathbf{F}_{a}, \mathbf{F}_{a}\right)_{L} \\
\underline{\left(\overline{\mathbf{A}}_{a}+\overline{\mathbf{S}}_{a}, 1,1,1\right)} L\end{array}$ & $\begin{array}{c}4 m_{a} n_{a} \\
4 m_{a} \\
2 m_{a}\left(n_{a}-1\right)\end{array}$ \\
\hline$a a^{\prime} T$ & $\left(\overline{\mathbf{F}}_{a}, 1,1, \overline{\mathbf{F}}_{a}\right)_{L}+\left(1, \overline{\mathbf{F}}_{a}, \overline{\mathbf{F}}_{a}, 1\right)_{L}$ & $2 m_{a} n_{a} \chi$ \\
\hline$a b U$ & $\begin{array}{c}\left(\mathbf{F}_{a}, 1,1,1 ; 1, \overline{\mathbf{F}}_{b}, 1,1\right)_{L}+\left(1, \mathbf{F}_{a}, 1,1 ; \overline{\mathbf{F}}_{b}, 1,1,1\right)_{L} \\
+\left(1,1, \mathbf{F}_{a}, 1 ; 1,1,1, \overline{\mathbf{F}}_{b}\right)_{L}+\left(1,1,1, \mathbf{F}_{a} ; 1,1, \overline{\mathbf{F}}_{b}, 1\right)_{L} \\
\left(\overline{\mathbf{F}}_{a}, 1,1,1 ; \mathbf{F}_{b}, 1,1,1\right)_{L}+\left(1, \overline{\mathbf{F}}_{a}, 1,1 ; 1, \mathbf{F}_{b}, 1,1\right)_{L} \\
+\left(1,1, \overline{\mathbf{F}}_{a}, 1 ; 1,1, \mathbf{F}_{b}, 1\right)_{L}+\left(1,1,1, \overline{\mathbf{F}}_{a} ; 1,1,1, \mathbf{F}_{b}\right)_{L} \\
\left(\overline{\mathbf{F}}_{a}, 1,1,1 ; \overline{\mathbf{F}}_{b}, 1,1,1\right)_{L}+\left(1, \overline{\mathbf{F}}_{a}, 1,1 ; 1, \overline{\mathbf{F}}_{b}, 1,1\right)_{L} \\
+\left(1,1, \overline{\mathbf{F}}_{a}, 1 ; 1,1, \overline{\mathbf{F}}_{b}, 1\right)_{L}+\left(1,1,1, \overline{\mathbf{F}}_{a} ; 1,1,1, \overline{\mathbf{F}}_{b}\right)_{L} \\
\left(\mathbf{F}_{a}, 1,1,1 ; 1, \mathbf{F}_{b}, 1,1\right)_{L}+\left(1, \mathbf{F}_{a}, 1,1 ; \mathbf{F}_{b}, 1,1,1\right)_{L} \\
+\left(1,1, \mathbf{F}_{a}, 1 ; 1,1,1, \mathbf{F}_{b}\right)_{L}+\left(1,1,1, \mathbf{F}_{a}: 1,1, \mathbf{F}_{b}, 1\right)_{L}\end{array}$ & $\begin{array}{l}2\left(n_{a} m_{b}-n_{b} m_{a}\right) \\
2\left(n_{a} m_{b}-n_{b} m_{a}\right) \\
2\left(n_{a} m_{b}+n_{b} m_{a}\right) \\
2\left(n_{a} m_{b}+n_{b} m_{a}\right)\end{array}$ \\
\hline $\begin{array}{l}a b T \\
a b^{\prime} T\end{array}$ & $\begin{aligned} & \left(\mathbf{F}_{a}, 1,1,1 ; 1,1, \overline{\mathbf{F}}_{b}, 1\right)_{L}+\left(1, \mathbf{F}_{a}, 1,1 ; 1,1,1, \overline{\mathbf{F}}_{b}\right)_{L} \\
+ & \left(1,1, \mathbf{F}_{a}, 1 ; \overline{\mathbf{F}}_{b}, 1,1,1,\right)_{L}+\left(1,1,1, \mathbf{F}_{a} ; 1, \overline{\mathbf{F}}_{b}, 1,1\right)_{L} \\
& \left(\overline{\mathbf{F}}_{a}, 1,1,1 ; 1,1,1, \overline{\mathbf{F}}_{b}\right)_{L}+\left(1, \overline{\mathbf{F}}_{a}, 1,1 ; 1,1, \overline{\mathbf{F}}_{b}, 1\right)_{L} \\
+ & \left(1,1, \overline{\mathbf{F}}_{a}, 1 ; 1, \overline{\mathbf{F}}_{b}, 1,1,\right)_{L}+\left(1,1,1, \overline{\mathbf{F}}_{a} ; \overline{\mathbf{F}}_{b}, 1,1,1\right)_{L}\end{aligned}$ & $\begin{array}{l}\left(n_{a} m_{b}-n_{b} m_{a}\right) \chi \\
\left(n_{a} m_{b}+n_{b} m_{a}\right) \chi\end{array}$ \\
\hline
\end{tabular}

Table 3: Generic chiral spectrum for $\mathbb{Z}_{2}$. " $U$ " labels identical configurations on $T_{2,3}$, " $T$ " denotes branes which are perpendicular on $T_{2,3}$.

\subsubsection{Example 2, $\mathbb{Z}_{2}$}

As we choose not to include anti-branes in our analysis, the SM gauge group can only be enclosed for the choice $\chi=1$ (cf. eq. (22)). Taking the aaa lattice and the minimal possible choice, namely

$$
\begin{array}{ll}
N_{1}=6, & \left(n_{1}, m_{1}\right)=(1,1), \\
N_{2}=4, & \left(n_{2}, m_{2}\right)=(1,0), \\
N_{3}=2, & \left(n_{3}, m_{3}\right)=(4,1),
\end{array}
$$

we obtain the gauge group $S U(3)^{4} \times S U(2)^{2} \times U(1)^{10}$. Herein, a sublety arises from the second stack of branes. The tadpole condition (22) has to be modified,

$$
\frac{N_{2}}{2}+\sum_{a \neq 2} n_{a} N_{a}=16 .
$$

This is in agreement with the fact that models containing only stacks of branes with $(n, m)=(1,0)$ are T-dual to the ones considered in $[8]$ for $\mathbb{Z}_{2}$ and [19] for $\mathbb{Z}_{3}$ leading to $U(16)^{2}$ and $S O(8)$ respectively. The modified equation (24) reflects the fact that the additional $\Omega \mathcal{R}$ symmetry of brane 2 
removes half of the degrees of freedom. Thus, the sector $1^{\prime} 2$ provides the anti-particles for the sector 12 (and similarly for 23 and $23^{\prime}$ ), whereas generically the sector $a^{\prime} b^{\prime}$ contains the anti-particles for the sector $a b$ (and similarly $a b^{\prime}$ is paired with $a^{\prime} b$ ). Therefore, in the sectors $13,13^{\prime}, \ldots$ we obtain an even number of generations transforming under the same gauge factors whereas in the sector 12 , there exists a single particle in the $\left(\mathbf{3}_{1}, \mathbf{2}_{2}\right)$ of $S U(3)_{1} \times S U(2)_{2}$. However, as the complete spectrum is symmetrically distributed among the gauge factors which are supported by a specific brane configuration, the total number of $\left(\mathbf{3}_{i}, \mathbf{2}_{j}\right)$ representations of all possible $S U(3)_{i} \times S U(2)_{j}$ $(i=1, \ldots 4 ; j=1,2)$ combinations is even. This can be easily seen by examining the chiral spectrum displayed in table 6. The model contains (at least) six non-anomalous $U(1)$ factors. A possible set of linear combinations is given by

$$
\begin{aligned}
& \tilde{Q}_{1}=Q_{1}+Q_{2}-3 Q_{7}-3 Q_{8}, \\
& \tilde{Q}_{2}=Q_{3}+Q_{4}-3 Q_{9}-3 Q_{10}, \\
& \tilde{Q}_{3}=Q_{1}-Q_{2}-3 Q_{5}, \\
& \tilde{Q}_{4}=Q_{3}-Q_{4}-3 Q_{6}, \\
& \tilde{Q}_{5}=-4 Q_{5}+Q_{7}-Q_{8}, \\
& \tilde{Q}_{6}=-4 Q_{6}+Q_{9}-Q_{10} .
\end{aligned}
$$

These charges are also listed in table 6. The remaining anomalous $U(1)$ 's should become massive by a generalized GS mechanism.

\section{Example 1}
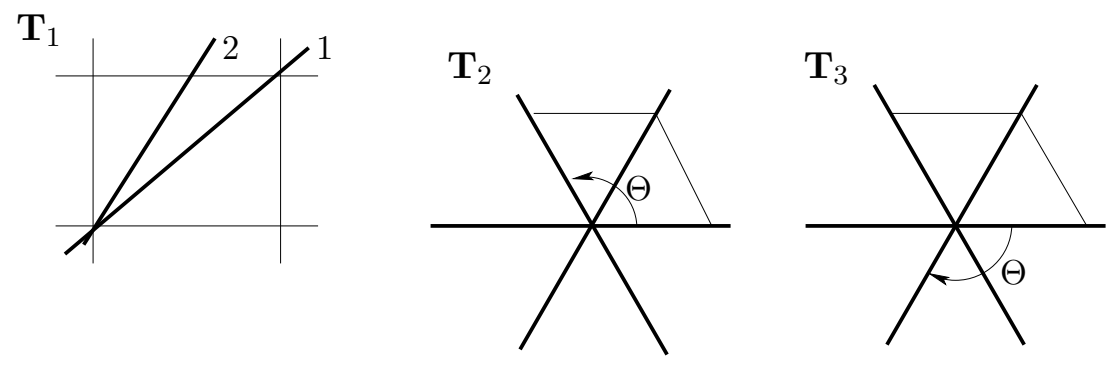

\section{Example 2}
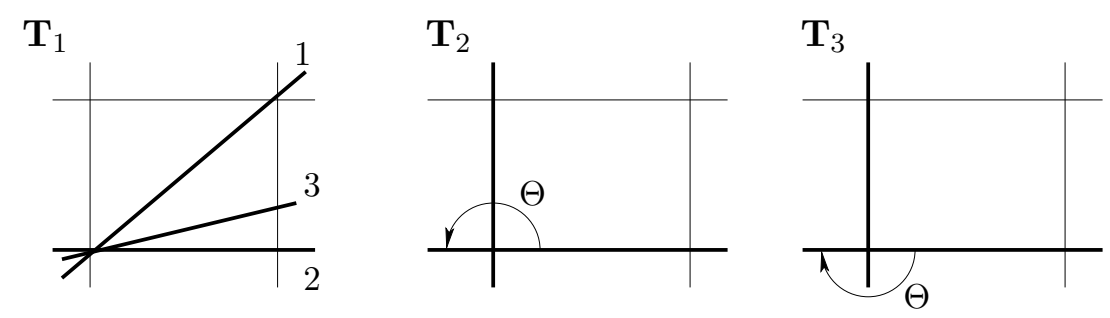

Figure 5: Brane configurations for the examples

\section{Conclusions}

We have constructed type IIA theories on $\Omega \mathcal{R} \times T^{2} \times T^{4} / \mathbb{Z}_{N}$. The resulting spectra are nonsupersymmetric. They contain a bunch of chiral fermions as well as tachyons transforming in the 
same representation of the gauge group. We have explicitly given examples for $\mathbb{Z}_{2,3}$. Excluding antiD-branes, for $\mathbb{Z}_{3}$ the maximal gauge group obtainable is $S U(3) \times U(1)$ if we require the presence of chiral fermions. The resulting spectrum is listed in table 2. We have also worked out an example for $\mathbb{Z}_{2}$ which encloses the standard model gauge group as well as several non-anomalous $U(1)$ factors. These $U(1)$ 's are displayed in equation (25) and the chiral spectrum is listed in table 6. However, in this framework we can neither obtain a three generation model nor give an obvious solution to the hierarchy problem. Both debilities could be avoided by combining a more general orientifold action $\Omega \tilde{\mathcal{R}}$ with non-vanishing background fields. Additionally, in such models tachyons would not necessarily carry the same gauge quantum numbers as the chiral fermions. We will pursue this further.

\section{Acknowledgments}

It is a pleasure to thank Ralph Blumenhagen, Boris Körs and Angel Uranga for valuable discussions. Further we acknowledge discussions with Jan O. Conrad, Hanno Klemm and Martin Walter. This work is supported by the European Commission RTN programs HPRN-CT-2000-00131, 00148 and 00152. 


\section{A Computation of one-loop diagrams}

The tadpole cancellation conditions are determined by computing those diagrams in the loop channel which correspond to RR-exchanges in the tree channel. As explained in section 2, the relevant contributions arise from the $\operatorname{NSNS}(-)^{F}$ sector for the Klein bottle, R for the Möbius strip and $\mathrm{NS}(-)^{F}$ for the Annulus. For the detailed calculation of the oscillator contributions in the loop channel, we refer to 21, 23, 41].

\section{A.1 Lattice contributions}

On a torus with radii $R_{1,2}$ only momenta along the $\Omega \mathcal{R}$ invariant direction and windings perpendicular to the former ones contribute. In the loop channel, the general contribution is given by

$$
\mathcal{L}^{R_{1}, R_{2}}[\alpha, \beta](t) \equiv\left(\sum_{m \in \mathbb{Z}} e^{-\alpha \pi t m^{2} / \rho_{1}}\right)\left(\sum_{n \in \mathbb{Z}} e^{-\beta \pi t n^{2} \rho_{2}}\right)
$$

where $\rho_{i}=R_{i}^{2} / \alpha^{\prime}$. Using Poisson resummation gives the general expression for the lattice contribution in the tree channel

$$
\tilde{\mathcal{L}}^{R_{1}, R_{2}}[\alpha, \beta](t) \equiv\left(\sum_{m \in \mathbb{Z}} e^{-\alpha \pi t m^{2} \rho_{1}}\right)\left(\sum_{n \in \mathbb{Z}} e^{-\beta \pi t n^{2} / \rho_{2}}\right) .
$$

(26) and (27) are related via

$$
\mathcal{L}^{R_{1}, R_{2}}[\alpha, \beta](t)=\operatorname{cl} \tilde{\mathcal{L}}^{R_{1}, R_{2}}\left[\frac{\kappa}{\alpha}, \frac{\kappa}{\beta}\right](l)
$$

with $c=\frac{\kappa}{\sqrt{\alpha \beta}} \frac{R_{1}}{R_{2}}, t=1 /(\kappa l)$ and $\kappa=4(2,8)$ for the Klein bottle (Annulus, Möbius strip). The results for the different tori are summarized in table 1 .

\section{B Computation of tree channel diagrams}

\section{B.1 Construction of crosscap states}

A comprehensive introduction into the boundary state approach to D branes is given in [30] and references therein. Appendix A of [38] contains the construction of crosscap states in related models.

\section{B.1.1 Oscillator part}

Crosscap states have to fulfill

$$
\left[X_{L, R}^{i}(\sigma, 0)-\Theta^{k} \mathcal{R} X_{R, L}^{i}(\sigma+\pi, 0)\right]\left|\Omega \mathcal{R} \Theta^{k}\right\rangle=0 .
$$

Inserting the mode expansion

$$
X^{i}(\sigma, \tau)=x^{i}+\frac{p_{L}^{i}}{2 \pi}(\tau+\sigma)+\frac{p_{R}^{i}}{2 \pi}(\tau-\sigma)+\frac{i}{2} \sum_{r} \frac{1}{r} \alpha_{r}^{i} e^{-i r(\tau+\sigma)}+\frac{i}{2} \sum_{s} \frac{1}{s} \tilde{\alpha}_{s}^{i} e^{-i s(\tau-\sigma)}
$$

gives the constraints in terms of bosonic oscillators

$$
\begin{aligned}
\left(\alpha_{n}^{\mu}+(-1)^{n} \tilde{\alpha}_{-n}^{\mu}\right)\left|\Omega \mathcal{R} \Theta^{k}, \eta\right\rangle & =0, \\
\left(\alpha_{r}^{i}+(-1)^{r} e^{2 \pi i k v_{i}} \tilde{\alpha}_{-r}^{\bar{i}}\right)\left|\Omega \mathcal{R} \Theta^{k}, \eta\right\rangle & =0, \\
\left(\alpha_{r}^{\bar{i}}+(-1)^{r} e^{-2 \pi i k v_{i}} \tilde{\alpha}_{-r}^{i}\right)\left|\Omega \mathcal{R} \Theta^{k}, \eta\right\rangle & =0 .
\end{aligned}
$$




\begin{tabular}{|c|c|c|c|c|c|c|}
\hline & & & \multicolumn{2}{|c|}{$\mathbb{Z}_{2,4}$} & \multicolumn{2}{|c|}{$\mathbb{Z}_{3,6}$} \\
& $\mathbf{a}$ & $\mathbf{b}$ & $\mathbf{A}$ & $\mathbf{B}$ & $\mathbf{A}$ & $\mathbf{B}$ \\
\hline \hline $\mathcal{L}^{\mathcal{K}}$ & $\mathcal{L}^{R_{1}, R_{2}}[1,1]$ & $\mathcal{L}^{R, R}\left[1 / \cos ^{2} \alpha, 4 \sin ^{2} \alpha\right]$ & $\mathcal{L}^{R, R}[1,1]$ & $\mathcal{L}^{R, R}[2,2]$ & $\mathcal{L}^{R, R}[4,3]$ & $\mathcal{L}^{R, R}[4 / 3,1]$ \\
\hline$\tilde{\mathcal{L}}^{\mathcal{K}}$ & $\tilde{\mathcal{L}}^{R_{1}, R_{2}}[4,4]$ & $\tilde{\mathcal{L}}^{R, R}\left[4 \cos ^{2} \alpha, 1 / \sin ^{2} \alpha\right]$ & $\tilde{\mathcal{L}}^{R, R}[4,4]$ & $\tilde{\mathcal{L}}^{R, R}[2,2]$ & $\tilde{\mathcal{L}}^{R, R}[1,4 / 3]$ & $\tilde{\mathcal{L}}^{R, R}[3,4]$ \\
\hline$c^{\mathcal{K}}$ & $4 R_{1} / R_{2}$ & $2 / \tan \alpha$ & 4 & 2 & $2 / \sqrt{3}$ & $2 \sqrt{3}$ \\
\hline \hline $\mathcal{L}^{\mathcal{A}}$ & $\mathcal{L}_{\mathbf{a}}^{\mathcal{A}}$ & $\mathcal{L}^{R, R}\left[2 / V_{\alpha}^{2}, 2 \sin ^{2}(2 \alpha) / V_{\alpha}^{2}\right]$ & $\mathcal{L}^{R, R}[2,2]$ & $\mathcal{L}^{R, R}[1,1]$ & $\mathcal{L}^{R, R}[2,3 / 2]$ & $\mathcal{L}^{R, R}[2 / 3,1 / 2]$ \\
\hline$\tilde{\mathcal{L}}^{\mathcal{A}}$ & $\tilde{\mathcal{L}}_{\mathbf{a}}^{\mathcal{A}}$ & $\tilde{\mathcal{L}}^{R, R}\left[V_{\alpha}^{2}, V_{\alpha}^{2} / \sin ^{2}(2 \alpha)\right]$ & $\tilde{\mathcal{L}}^{R, R}[1,1]$ & $\tilde{\mathcal{L}}^{R, R}[2,2]$ & $\tilde{\mathcal{L}}^{R, R}[1,4 / 3]$ & $\tilde{\mathcal{L}}^{R, R}[3,4]$ \\
\hline$c^{\mathcal{A}}$ & $V^{2} /\left(R_{1} R_{2}\right)$ & $V_{\alpha}^{2} / \sin (2 \alpha)$ & 1 & 2 & $2 / \sqrt{3}$ & $2 \sqrt{3}$ \\
\hline \hline $\mathcal{L}^{\mathcal{M}}$ & $\mathcal{L}^{R_{1}, R_{2}}[2,2]$ & $\mathcal{L}^{R, R}\left[1 /\left(2 \cos ^{2} \alpha\right), 8 \sin ^{2} \alpha\right]$ & $\mathcal{L}^{R, R}[2,2]$ & $\mathcal{L}^{R, R}[1,4]$ & $\mathcal{L}^{R, R}[2,6]$ & $\mathcal{L}^{R, R}[2 / 3,2]$ \\
\hline$\tilde{\mathcal{L}}^{\mathcal{M}}$ & $\tilde{\mathcal{L}}^{R,}, R_{2}[4,4]$ & $\left.\tilde{\mathcal{L}}^{R, R}\left[16 \cos ^{2} \alpha\right), 1 / \sin ^{2} \alpha\right]$ & $\mathcal{L}^{R, R}[4,4]$ & $\mathcal{L}^{R, R}[8,2]$ & $\mathcal{L}^{R, R}[4,4 / 3]$ & $\mathcal{L}^{R, R}[12,4]$ \\
\hline$c^{\mathcal{M}}$ & $4 R_{1} / R_{2}$ & $4 / \tan \alpha$ & 4 & 4 & $4 / \sqrt{3}$ & $4 \sqrt{3}$ \\
\hline
\end{tabular}

Table 4: The different lattice contributions. $V_{\alpha}=\sqrt{n_{a}^{2}+m_{a}^{2}+2 n_{a} m_{a} \cos (2 \alpha)}$. The definitions of $\mathcal{L}_{\mathbf{a}}^{\mathcal{A}}$ and $V$ are given in the text in section 3.1 .

The constraints for the fermionic oscillators are $(\eta= \pm 1)$

$$
\begin{aligned}
\left(\psi_{r}^{\mu}+i \eta e^{-i \pi r} \tilde{\psi}_{-r}^{\mu}\right)\left|\Omega \mathcal{R} \Theta^{k}, \eta\right\rangle & =0, \\
\left(\psi_{r}^{i}+i \eta e^{-i \pi r} e^{2 \pi i k v_{i}} \tilde{\psi}_{-r}^{\bar{i}}\right)\left|\Omega \mathcal{R} \Theta^{k}, \eta\right\rangle & =0 \\
\left(\psi_{r}^{\bar{i}}+i \eta e^{-i \pi r} e^{-2 \pi i k v_{i}} \tilde{\psi}_{-r}^{i}\right)\left|\Omega \mathcal{R} \Theta^{k}, \eta\right\rangle & =0 .
\end{aligned}
$$

A solution is provided by

$$
\begin{aligned}
\left|\Omega \mathcal{R} \Theta^{k}, \eta\right\rangle & =\mathcal{N}_{C} \exp \left\{-\sum_{n} \frac{(-1)^{n}}{n} \alpha_{-n}^{\mu} \tilde{\alpha}_{-n}^{\mu}-\sum_{i \in\{1,2,3\}} \sum_{n} \frac{(-1)^{n}}{n} e^{-2 \pi i k v_{i}} \alpha_{-n}^{i} \tilde{\alpha}_{-n}^{i}\right. \\
& -\sum_{\bar{i} \in\{\overline{1}, \overline{2}, \overline{3}\}} \sum_{n} \frac{(-1)^{n}}{n} e^{2 \pi i k v_{i}} \alpha_{-n}^{\bar{i}} \tilde{\alpha}_{-n}^{\bar{i}}-i \eta \sum_{r} e^{-i \pi r} \psi_{-r}^{\mu} \tilde{\psi}_{-r}^{\mu} \\
& \left.-i \eta \sum_{i \in\{1,2,3\}} \sum_{r} e^{-i \pi r} e^{-2 \pi i k v_{i}} \psi_{-r}^{i} \tilde{\psi}_{-r}^{i}-i \eta \sum_{\bar{i} \in\{\overline{1}, \overline{2}, \overline{3}\}} \sum_{r} e^{-i \pi r} e^{2 \pi i k v_{i}} \psi_{-r}^{\bar{i}} \tilde{\psi}_{-r}^{\bar{i}}\right\}|0, \eta\rangle
\end{aligned}
$$

where $r \in \mathbb{Z}\left(\mathbb{Z}+\frac{1}{2}\right)$ in the RR (NSNS) sector. GSO-invariant states are given by

$$
\begin{aligned}
\left|\Omega \mathcal{R} \Theta^{k}\right\rangle & =\left|\Omega \mathcal{R} \Theta^{k}\right\rangle_{N S N S}+\left|\Omega \mathcal{R} \Theta^{k}\right\rangle_{R R} \\
\left|\Omega \mathcal{R} \Theta^{k}\right\rangle_{N S N S} & =\left|\Omega \mathcal{R} \Theta^{k},+\right\rangle_{N S N S}-\left|\Omega \mathcal{R} \Theta^{k},-\right\rangle_{N S N S}, \\
\left|\Omega \mathcal{R} \Theta^{k}\right\rangle_{R R} & =\left|\Omega \mathcal{R} \Theta^{k},+\right\rangle_{R R}-i\left|\Omega \mathcal{R} \Theta^{k},-\right\rangle_{R R} .
\end{aligned}
$$


The total crosscap state has to be invariant w.r.t. the orbifold group (i.e. it contains the "complete projector'):

$$
|C\rangle=\sum_{k=0}^{N-1}\left|\Omega \mathcal{R} \Theta^{k}\right\rangle .
$$

\section{B.1.2 Lattice part}

From (29) we also obtain

$$
\left(x^{i}-e^{2 \pi i k v_{i}}\left[x^{\bar{i}}+\frac{1}{2}\left(p_{L}^{\bar{i}}-p_{R}^{\bar{i}}\right)\right]\right)\left|\Omega \mathcal{R} \Theta^{k}\right\rangle=0
$$

by inserting the mode expansion (30). From this we can read off that the crosscap state $\left|\Omega \mathcal{R} \Theta^{k}\right\rangle$ is confined to a line on $T_{i}$ which is tilted by the angle $-\pi k v_{i}$ w.r.t. the real axis. Finally, conditions on the momenta arise:

$$
\begin{aligned}
p^{\mu}\left|\Omega \mathcal{R} \Theta^{k}\right\rangle & =0, \\
\left(p_{L}^{i}+e^{2 \pi i k v_{i}} p_{R}^{\bar{i}}\right)\left|\Omega \mathcal{R} \Theta^{k}\right\rangle & =0, \\
\left(p_{R}^{i}+e^{2 \pi i k v_{i}} p_{L}^{\bar{i}}\right)\left|\Omega \mathcal{R} \Theta^{k}\right\rangle & =0 .
\end{aligned}
$$

Inserting $p_{L, R}=P \pm \alpha^{\prime} W$ for the compact momenta, (37) indicates that on each $T_{i}$, there are Kaluza-Klein momenta perpendicular and windings parallel to the position of the crosscap state.

\section{B.2 Boundary states}

Similarly to a crosscap state, the boundary state for a D-brane at angle $\pi \varphi$ on $T_{1}$ w.r.t. the $x^{4}$ axis is given by

$$
\begin{aligned}
\left|\varphi, \Theta^{k} ; \eta\right\rangle= & \mathcal{N}_{B} \quad \exp \left\{-\sum_{n} \frac{1}{n} \alpha_{-n}^{\mu} \tilde{\alpha}_{-n}^{\mu}-\sum_{n} \frac{1}{n} e^{2 \pi i \varphi} \alpha_{-n}^{1} \tilde{\alpha}_{-n}^{1}-\sum_{i \in\{2,3\}} \sum_{n} \frac{1}{n} e^{-2 \pi i k v_{i}} \alpha_{-n}^{i} \tilde{\alpha}_{-n}^{i}\right. \\
& -\sum_{n} \frac{1}{n} e^{-2 \pi i \varphi} \alpha_{-n}^{\overline{1}} \tilde{\alpha}_{-n}^{\overline{1}}-\sum_{\bar{i} \in\{\overline{2}, \overline{3}\}} \sum_{n} \frac{1}{n} e^{2 \pi i k v_{i}} \alpha_{-n}^{\bar{i}} \tilde{\alpha}_{-n}^{\bar{i}} \\
& -\quad i \eta \sum_{r} \psi_{-r}^{\mu} \tilde{\psi}_{-r}^{\mu}-i \eta \sum_{r} e^{2 \pi i \varphi} \psi_{-r}^{1} \tilde{\psi}_{-r}^{1}-i \eta \sum_{i \in\{2,3\}} \sum_{r} e^{-2 \pi i k v_{i}} \psi_{-r}^{i} \tilde{\psi}_{-r}^{i} \\
& \left.-\quad i \eta \sum_{r} e^{-2 \pi i \varphi} \psi_{-r}^{\overline{1}} \tilde{\psi}_{-r}^{\overline{1}}-i \eta \sum_{\bar{i} \in\{\overline{2}, \overline{3}\}} \sum_{r} e^{2 \pi i k v_{i}} \psi_{-r}^{\bar{i}} \tilde{\psi}_{-r}^{\bar{i}}\right\}|0, \eta\rangle
\end{aligned}
$$

Using the analogous equations to (34), the GSO-ivariant boundary state is given by

$$
\left|B_{\varphi}\right\rangle=\sum_{k=0}^{N-1}\left|\varphi, \Theta^{k}\right\rangle .
$$

Discrete momenta exist in the compact directions perpendicular to the position of the boundary state while windings are parallel. 


\section{B.3 Tree channel amplitudes}

Using equation (35) and (39), the tree channel amplitudes read

$$
\begin{aligned}
\tilde{\mathcal{K}}_{\text {total }} & =\tilde{\mathcal{K}}_{R R}+\tilde{\mathcal{K}}_{N S N S}=\int_{0}^{\infty} d l\left\langle C\left|e^{-2 \pi l H_{c l}}\right| C\right\rangle \\
\tilde{\mathcal{A}}_{\text {total }} & =\tilde{\mathcal{A}}_{R R}+\tilde{\mathcal{A}}_{N S N S}=\int_{0}^{\infty} d l \sum_{\varphi, \varphi^{\prime}}\left\langle B_{\varphi}\left|e^{-2 \pi l H_{c l}}\right| B_{\varphi^{\prime}}\right\rangle \\
\tilde{\mathcal{M}}_{\text {total }} & =\tilde{\mathcal{M}}_{R R}+\tilde{\mathcal{M}}_{N S N S}=\int_{0}^{\infty} d l \sum_{\varphi}\left(\left\langle C\left|e^{-2 \pi l H_{c l}}\right| B_{\varphi}\right\rangle+\text { h.c. }\right)
\end{aligned}
$$

As we only need to compute the RR exchange, we will use the abbreviation $\tilde{\mathcal{K}} \equiv \tilde{\mathcal{K}}_{R R}$ etc. The normalizations $\mathcal{N}_{C}, \mathcal{N}_{B}$ are determined from the Klein bottle and Annulus amplitude via worldsheet duality. The following equation holds

$$
\left\langle\Omega \mathcal{R} \Theta^{k}\left|e^{-2 \pi l H_{c l}}\right| \Omega \mathcal{R} \Theta^{k^{\prime}}\right\rangle= \begin{cases}\mathcal{N}_{C}^{2} \tilde{\mathcal{K}}^{(0)} \tilde{\mathcal{L}}_{1} \tilde{\mathcal{L}}_{2} \tilde{\mathcal{L}}_{3} & \text { for } k=k^{\prime} \\ \prod_{i=1}^{2}\left[-2 \sin \left(\pi v_{i}\left(k-k^{\prime}\right)\right)\right] \mathcal{N}_{C}^{2} \tilde{\mathcal{K}}^{\left(k-k^{\prime}\right)} \tilde{\mathcal{L}}_{1} & \text { for } k \neq k^{\prime}\end{cases}
$$

where $\tilde{\mathcal{K}}^{\left(k-k^{\prime}\right)} \equiv \tilde{\mathcal{K}}^{\left(k-k^{\prime}\right)}(l)$ contains the oscillator contribution (for notation see [21, 23]) and $\tilde{\mathcal{L}}_{i} \equiv$ $\tilde{\mathcal{L}}_{i}(l)$ denotes the lattice contribution for the torus $T_{i}$. For $\mathbb{Z}_{2,3}$, all $\left|\Omega \mathcal{R} \Theta^{k}\right\rangle$ are extended along (diagonal to) the axes of $T_{2,3}$ in the case of the $\mathbf{A}(\mathbf{B})$ lattice. Since branes lie on top of O-planes on $T_{2,3}$ in our models, the positions of the O-planes can be read off from figure 5 . All $\left|\Omega \mathcal{R} \Theta^{k}\right\rangle$ have the same relative orientation w.r.t. the tori $T_{2,3}$, hence they all provide the same lattice contribution. All choices AA, AB, BB lead to consistent models. The situation is different for $\mathbb{Z}_{4,6}$. In these models, all $\left|\Omega \mathcal{R} \Theta^{2 k}\right\rangle$ have the same orientation w.r.t. the lattice while all $\left|\Omega \mathcal{R} \Theta^{2 k+1}\right\rangle$ have the other possible one. $\left|\Omega \mathcal{R} \Theta^{2 k}\right\rangle$ on the lattice $\mathbf{A}$ gives the same contribution as $\left|\Omega \mathcal{R} \Theta^{2 k+1}\right\rangle$ on $\mathbf{B}$ and vice versa,

$$
\left\langle\Omega \mathcal{R} \Theta^{2 k}\left|e^{-2 \pi l H_{c l}}\right| \Omega \mathcal{R} \Theta^{2 k}\right\rangle+\left\langle\Omega \mathcal{R} \Theta^{2 k+1}\left|e^{-2 \pi l H_{c l}}\right| \Omega \mathcal{R} \Theta^{2 k+1}\right\rangle \longrightarrow\left\{\begin{array}{cc}
2 \tilde{\mathcal{L}}_{\mathbf{A}} \tilde{\mathcal{L}}_{\mathbf{B}} & \text { for } \mathbf{A B} \\
\tilde{\mathcal{L}}_{\mathbf{A}}^{2}+\tilde{\mathcal{L}}_{\mathbf{B}}^{2} & \text { for } \mathbf{A A} / \mathbf{B B}
\end{array}\right.
$$

By modular transformation from the loop channel, one recovers the correct result for $\mathbf{A B}$. But for AA or BB, the loop channel amplitude gives $4 \tilde{\mathcal{L}}_{\mathbf{A}}^{2}+\tilde{\mathcal{L}}_{\mathbf{B}}^{2}$ for $\mathbb{Z}_{4}\left(\tilde{\mathcal{L}}_{\mathbf{A}}^{2}+9 \tilde{\mathcal{L}}_{\mathbf{B}}^{2}\right.$ for $\left.\mathbb{Z}_{6}\right)$ as can be read off from table 4 . This means that only the AB-lattice is consistent with worldsheet duality.

\section{Spectra}




\section{C.1 Closed string spectra}

\begin{tabular}{|c|c|c|c|c|c|}
\hline & & & & \multicolumn{2}{|c|}{ Closed string spectrum for $\mathbb{Z}_{4}$} \\
\hline \multicolumn{4}{|c|}{ Closed string spectrum for $\mathbb{Z}_{2}$} & lattice & $\mathrm{AB}$ \\
\hline twist-sector & $\mathbf{A A}$ & $\mathrm{AB}$ & $\mathrm{BB}$ & untwisted & SUGRA $+8 \mathrm{C}+3 \mathrm{~V}$ \\
\hline untwisted & \multicolumn{3}{|c|}{$\mathrm{SUGRA}+11 \mathrm{C}+4 \mathrm{~V}$} & $\theta_{1}+\theta_{1}^{3}$ & $16 C$ \\
\hline$\theta_{1}$ & $32 \mathrm{C}$ & $28 \Gamma$ & 260 & $\theta_{1}^{2}$ & $19 C+1 V$ \\
\hline \multirow{2}{*}{\multicolumn{4}{|c|}{ Closed spectrum of $\mathbb{Z}_{3}$}} & \multicolumn{2}{|c|}{ Closed string spectrum for $\mathbb{Z}_{6}$} \\
\hline & & & & untwisted & $\mathrm{SUGRA}+8 \mathrm{C}+3 \mathrm{~V}$ \\
\hline untwisted & \multicolumn{3}{|c|}{$\mathrm{SUGRA}+8 \mathrm{C}+3 \mathrm{~V}$} & $\theta^{5}+\theta^{5}$ & $4 \mathrm{C}$ \\
\hline \multirow[t]{2}{*}{$\theta_{1}+\theta_{1}^{2}$} & \multirow[t]{2}{*}{$28 \mathrm{C}+8 \mathrm{~V}$} & \multirow[t]{2}{*}{$30 \mathrm{C}+6 \mathrm{~V}$} & \multirow[t]{2}{*}{$36 \mathrm{C}$} & $\frac{\theta_{1}+\theta_{1}}{\Omega^{2}}$ & 40 \\
\hline & & & & $\frac{\sigma_{1}+0}{\theta^{3}}$ & $\frac{10 \mathrm{U}+2 \mathrm{~V}}{11 \mathrm{C}+1 \mathrm{~V}}$ \\
\hline
\end{tabular}

\section{C.2 Open string spectra}

The multiplicity of open string states is determined by the number of intersections of brane $a$ and $b$ on $T_{1}$ in terms of their wrapping numbers,

$$
I_{a b}=n_{a} m_{b}-m_{a} n_{b},
$$

as well as by the number of intersections $\chi$ on $T_{2,3}$. An $\mathbf{A}$ type torus contributes a factor of one, whereas a $\mathbf{B}$ type torus contributes a factor of $2(3)$ in the case of $\mathbb{Z}_{2,4}\left(\mathbb{Z}_{3,6}\right)$.

For mirror branes, we have to distinguish between $\Omega \mathcal{R}$ invariant intersections and those which form pairs under $\Omega \mathcal{R}$ :

$$
\begin{aligned}
I_{a^{\prime} a}^{\Omega \mathcal{R}} & \equiv\left\{\begin{array}{cc}
2 m_{a} & \mathbf{a} \\
m_{a}-n_{a} & \mathbf{b}
\end{array}\right. \\
I_{a^{\prime} a}-I_{a^{\prime} a}^{\Omega \mathcal{R}} & =\left\{\begin{array}{cc}
2 m_{a}\left(n_{a}-1\right) & \mathbf{a} \\
{\left[m_{a}\left(m_{a}-1\right)-n_{a}\left(n_{a}-1\right)\right]} & \mathbf{b}
\end{array}\right.
\end{aligned}
$$

\section{C.2.1 Fermionic states}

The sectors where the branes are parallel on the first torus are non-chiral. In the sector with $\Delta \varphi \neq 0$ and $\frac{k}{N} \neq 0$, the left-handed fermion is massless while the would-be right-handed one becomes massive with $\alpha^{\prime} m^{2}=\Delta \varphi$. For $\mathbb{Z}_{2,4,6}$, additional chiral fermions arise from strings stretching between mirror branes with $\frac{k}{N}=0$ because the left handed R-states are even under the $\mathbb{Z}_{2}$ symmetry while the right handed ones are odd and thus are subject to a different orbifold projection. 
Fermionic states on $T^{2} \times T^{4} / \mathbb{Z}_{N}$

\begin{tabular}{|c|c||l|c|c||c|}
\hline \hline on $T^{2}$ & on $T^{4} / \mathbb{Z}_{2}$ & state & mass & chirality & $\mathbb{Z}_{2}$ \\
\hline \hline$\Delta \varphi=0$ & $\frac{k}{N}=0$ & $|0\rangle_{R}$ & 0 & $L$ & + \\
\hline & & $\psi_{0}^{0} \psi_{0}^{1}|0\rangle_{R}$ & 0 & $R$ & + \\
\hline & & $\psi_{0}^{0} \psi_{0}^{2}|0\rangle_{R}$ & 0 & $R$ & - \\
\hline & & $\psi_{0}^{0} \psi_{0}^{3}|0\rangle_{R}$ & 0 & $R$ & - \\
\hline & & $\psi_{0}^{1} \psi_{0}^{2}|0\rangle_{R}$ & 0 & $L$ & - \\
\hline & & $\psi_{0}^{1} \psi_{0}^{3}|0\rangle_{R}$ & 0 & $L$ & - \\
\hline & & $\psi_{0}^{2} \psi_{0}^{3}|0\rangle_{R}$ & 0 & $L$ & + \\
\hline & & $\psi_{0}^{0} \psi_{0}^{1} \psi_{0}^{2} \psi_{0}^{3}|0\rangle_{R}$ & 0 & $R$ & + \\
\hline \hline$\Delta \varphi=0$ & $0<\frac{k}{N} \leqslant \frac{1}{2}$ & $|0\rangle_{R}$ & 0 & $L$ & + \\
\hline \hline$\Delta \varphi \neq 0$ & & $\psi_{0}^{0} \psi_{0}^{1}|0\rangle_{R}$ & 0 & $R$ & + \\
\hline & & $|0\rangle_{R}$ & 0 & $L$ & + \\
\hline & & $\psi_{0}^{0} \psi_{0}^{2}|0\rangle_{R}$ & 0 & $R$ & - \\
\hline & & $\psi_{0}^{0} \psi_{0}^{3}|0\rangle_{R}$ & 0 & $R$ & - \\
\hline \hline$\Delta \varphi \neq 0$ & $0<\frac{k}{N} \leqslant \frac{1}{2}$ & $|0\rangle_{R}$ & 0 & $L$ & + \\
\hline & & $\psi_{0}^{0} \psi_{-\Delta \varphi}^{1}|0\rangle_{R}$ & $\Delta \varphi$ & $(R)$ & + \\
\hline
\end{tabular}

Table 5: Fermionic ground states for the open string sector 
Chiral spectrum, Ex. 2, Part 1

\begin{tabular}{|c|c|c|c|c|c|c|c|c|c|c|c|c|c|c|c|c|c|c|}
\hline & rep. & mult & $Q_{1}$ & $Q_{2}$ & $Q_{3}$ & $Q_{4}$ & $Q_{5}$ & $Q_{6}$ & $Q_{7}$ & $Q_{8}$ & $Q_{9}$ & $Q_{10}$ & $\tilde{Q}_{1}$ & $\tilde{Q}_{2}$ & $\tilde{Q}_{3}$ & $\tilde{Q}_{4}$ & $\tilde{Q}_{5}$ & $\tilde{Q}_{6}$ \\
\hline \multirow[t]{6}{*}{$11^{\prime} U$} & $\|(\mathbf{3}, \mathbf{3}, 1,1 ; 1,1)$ & 4 & 1 & 1 & 0 & 0 & 0 & 0 & 0 & 0 & 0 & 0 & 2 & 0 & 0 & 0 & 0 & 0 \\
\hline & $\|(1,1, \mathbf{3}, \mathbf{3} ; 1,1)$ & 4 & 0 & 0 & 1 & 1 & 0 & 0 & 0 & 0 & 0 & 0 & 0 & 2 & 0 & 0 & 0 & 0 \\
\hline & $\|(\mathbf{3}, 1,1,1 ; 1,1)$ & 4 & -2 & 0 & 0 & 0 & 0 & 0 & 0 & 0 & 0 & 0 & -2 & 0 & -2 & 0 & 0 & 0 \\
\hline & $(1, \mathbf{3}, 1,1 ; 1,1)$ & 4 & 0 & -2 & 0 & 0 & 0 & 0 & 0 & 0 & 0 & 0 & -2 & 0 & 2 & 0 & 0 & 0 \\
\hline & $\|(1,1,3,1 ; 1,1)$ & 4 & 0 & 0 & -2 & 0 & 0 & 0 & 0 & 0 & 0 & 0 & 0 & -2 & 0 & -2 & 0 & 0 \\
\hline & $(1,1,1, \mathbf{3} ; 1,1)$ & 4 & 0 & 0 & 0 & -2 & 0 & 0 & 0 & 0 & 0 & 0 & 0 & -2 & 0 & 2 & 0 & 0 \\
\hline \multirow[t]{2}{*}{$11^{\prime} T$} & $\|(\overline{\mathbf{3}}, 1,1, \overline{\mathbf{3}} ; 1,1)$ & 2 & -1 & 0 & 0 & -1 & 0 & 0 & 0 & 0 & 0 & 0 & -1 & -1 & -1 & 1 & 0 & 0 \\
\hline & $(1, \overline{\mathbf{3}}, \overline{\mathbf{3}}, 1 ; 1,1)$ & 2 & 0 & -1 & -1 & 0 & 0 & 0 & 0 & 0 & 0 & 0 & -1 & -1 & 1 & -1 & 0 & 0 \\
\hline \multirow[t]{8}{*}{$12 U$} & $(\mathbf{3}, 1,1,1 ; \overline{\mathbf{2}}, 1)$ & 2 & 1 & 0 & 0 & 0 & -1 & 0 & 0 & 0 & 0 & 0 & 1 & 0 & 4 & 0 & 4 & 0 \\
\hline & $(\overline{\mathbf{3}}, 1,1,1 ; \overline{\mathbf{2}}, 1)$ & 2 & -1 & 0 & 0 & 0 & -1 & 0 & 0 & 0 & 0 & 0 & -1 & 0 & 2 & 0 & 4 & 0 \\
\hline & $(1, \mathbf{3}, 1,1 ; \mathbf{2}, 1)$ & 2 & 0 & 1 & 0 & 0 & 1 & 0 & 0 & 0 & 0 & 0 & 1 & 0 & -4 & 0 & -4 & 0 \\
\hline & $(1, \overline{\mathbf{3}}, 1,1 ; \mathbf{2}, 1)$ & 2 & 0 & -1 & 0 & 0 & 1 & 0 & 0 & 0 & 0 & 0 & -1 & 0 & -2 & 0 & -4 & 0 \\
\hline & $(1,1, \mathbf{3}, 1 ; 1, \overline{\mathbf{2}})$ & 2 & 0 & 0 & 1 & 0 & 0 & -1 & 0 & 0 & 0 & 0 & 0 & 1 & 0 & 4 & 0 & 4 \\
\hline & $(1,1, \overline{\mathbf{3}}, 1 ; 1, \overline{\mathbf{2}})$ & 2 & 0 & 0 & -1 & 0 & 0 & -1 & 0 & 0 & 0 & 0 & 0 & -1 & 0 & 2 & 0 & 4 \\
\hline & $(1,1,1, \mathbf{3} ; 1, \mathbf{2})$ & 2 & 0 & 0 & 0 & 1 & 0 & 1 & 0 & 0 & 0 & 0 & 0 & 1 & 0 & -4 & 0 & -4 \\
\hline & $(1,1,1, \overline{\mathbf{3}} ; 1, \mathbf{2})$ & 2 & 0 & 0 & 0 & -1 & 0 & 1 & 0 & 0 & 0 & 0 & 0 & -1 & 0 & -2 & 0 & -4 \\
\hline \multirow[t]{4}{*}{$12 T$} & $(\overline{\mathbf{3}}, 1,1,1 ; 1, \mathbf{2})$ & 1 & -1 & 0 & 0 & 0 & 0 & 1 & 0 & 0 & 0 & 0 & -1 & 0 & -1 & -3 & 0 & -4 \\
\hline & $(1, \overline{\mathbf{3}}, 1,1 ; 1, \overline{\mathbf{2}})$ & 1 & 0 & -1 & 0 & 0 & 0 & -1 & 0 & 0 & 0 & 0 & -1 & 0 & 1 & 3 & 0 & 4 \\
\hline & $(1,1, \overline{\mathbf{3}}, 1 ; \mathbf{2}, 1)$ & 1 & 0 & 0 & -1 & 0 & 1 & 0 & 0 & 0 & 0 & 0 & 0 & -1 & -3 & -1 & -4 & 0 \\
\hline & $(1,1,1, \overline{\mathbf{3}} ; \overline{\mathbf{2}}, 1)$ & 1 & 0 & 0 & 0 & -1 & -1 & 0 & 0 & 0 & 0 & 0 & 0 & -1 & 3 & 1 & 4 & 0 \\
\hline \multirow[t]{8}{*}{$13 U$} & $(\mathbf{3}, 1,1,1 ; 1,1)$ & 6 & 1 & 0 & 0 & 0 & 0 & 0 & -1 & 0 & 0 & 0 & 4 & 0 & 1 & 0 & -1 & 0 \\
\hline & $(\overline{\mathbf{3}}, 1,1,1 ; 1,1)$ & 6 & -1 & 0 & 0 & 0 & 0 & 0 & 0 & 1 & 0 & 0 & -4 & 0 & -1 & 0 & -1 & 0 \\
\hline & $(1, \mathbf{3}, 1,1 ; 1,1)$ & 6 & 0 & 1 & 0 & 0 & 0 & 0 & 0 & -1 & 0 & 0 & 4 & 0 & -1 & 0 & 1 & 0 \\
\hline & $(1, \overline{\mathbf{3}}, 1,1 ; 1,1)$ & 6 & 0 & -1 & 0 & 0 & 0 & 0 & 1 & 0 & 0 & 0 & -4 & 0 & 1 & 0 & 1 & 0 \\
\hline & $(1,1, \mathbf{3}, 1 ; 1,1)$ & 6 & 0 & 0 & 1 & 0 & 0 & 0 & 0 & 0 & -1 & 0 & 0 & 4 & 0 & 1 & 0 & -1 \\
\hline & $(1,1, \overline{\mathbf{3}}, 1 ; 1,1)$ & 6 & 0 & 0 & -1 & 0 & 0 & 0 & 0 & 0 & 0 & 1 & 0 & -4 & 0 & -1 & 0 & -1 \\
\hline & $(1,1,1, \mathbf{3} ; 1,1)$ & 6 & 0 & 0 & 0 & 1 & 0 & 0 & 0 & 0 & 0 & -1 & 0 & 4 & 0 & -1 & 0 & 1 \\
\hline & $(1,1,1, \overline{\mathbf{3}} ; 1,1)$ & 6 & 0 & 0 & 0 & -1 & 0 & 0 & 0 & 0 & 1 & 0 & 0 & -4 & 0 & 1 & 0 & 1 \\
\hline \multirow[t]{8}{*}{$13^{\prime} U$} & $(\mathbf{3}, 1,1,1 ; 1,1)$ & 10 & 1 & 0 & 0 & 0 & 0 & 0 & 0 & 1 & 0 & 0 & -2 & 0 & 1 & 0 & -1 & 0 \\
\hline & $(\overline{\mathbf{3}}, 1,1,1 ; 1,1)$ & 10 & -1 & 0 & 0 & 0 & 0 & 0 & -1 & 0 & 0 & 0 & 2 & 0 & -1 & 0 & -1 & 0 \\
\hline & $(1, \mathbf{3}, 1,1 ; 1,1)$ & 10 & 0 & 1 & 0 & 0 & 0 & 0 & 1 & 0 & 0 & 0 & -2 & 0 & -1 & 0 & 1 & 0 \\
\hline & $(1, \overline{\mathbf{3}}, 1,1 ; 1,1)$ & 10 & 0 & -1 & 0 & 0 & 0 & 0 & 0 & -1 & 0 & 0 & 2 & 0 & 1 & 0 & 1 & 0 \\
\hline & $(1,1,3,1 ; 1,1)$ & 10 & 0 & 0 & 1 & 0 & 0 & 0 & 0 & 0 & 0 & 1 & 0 & -2 & 0 & 1 & 0 & -1 \\
\hline & $(1,1, \overline{\mathbf{3}}, 1 ; 1,1)$ & 10 & 0 & 0 & -1 & 0 & 0 & 0 & 0 & 0 & -1 & 0 & 0 & 2 & 0 & -1 & 0 & -1 \\
\hline & $(1,1,1, \mathbf{3} ; 1,1)$ & 10 & 0 & 0 & 0 & 1 & 0 & 0 & 0 & 0 & 1 & 0 & 0 & -2 & 0 & -1 & 0 & 1 \\
\hline & $(1,1,1, \overline{\mathbf{3}} ; 1,1)$ & 10 & 0 & 0 & 0 & -1 & 0 & 0 & 0 & 0 & 0 & -1 & 0 & 2 & 0 & 1 & 0 & 1 \\
\hline
\end{tabular}


Chiral spectrum, Ex. 2, Part 2

\begin{tabular}{|c|c|c|c|c|c|c|c|c|c|c|c|c|c|c|c|c|c|c|}
\hline & rep. & mult & $Q_{1}$ & $Q_{2}$ & $Q_{3}$ & $Q_{4}$ & $Q_{5}$ & $Q_{6}$ & $Q_{7}$ & $Q_{8}$ & $Q_{9}$ & $Q_{10}$ & $\tilde{Q}_{1}$ & $\tilde{Q}_{2}$ & $\overline{\tilde{Q}_{3}}$ & $\overline{\tilde{Q}_{4}}$ & $\overline{\tilde{Q}_{5}}$ & $\overline{\overline{Q_{Q}}}$ \\
\hline \multirow[t]{4}{*}{$13 T$} & $\mid(\overline{\mathbf{3}}, 1,1,1 ; 1,1)$ & 3 & -1 & 0 & 0 & 0 & 0 & 0 & 0 & 0 & 1 & 0 & -1 & -3 & -1 & 0 & 0 & 1 \\
\hline & $\|(1, \overline{\mathbf{3}}, 1,1 ; 1,1)$ & 3 & 0 & -1 & 0 & 0 & 0 & 0 & 0 & 0 & 0 & 1 & -1 & -3 & 1 & 0 & 0 & -1 \\
\hline & $\|(1,1, \overline{\mathbf{3}}, 1 ; 1,1)$ & 3 & 0 & 0 & -1 & 0 & 0 & 0 & 1 & 0 & 0 & 0 & -3 & -1 & 0 & -1 & 1 & 0 \\
\hline & $\|(1,1,1, \overline{\mathbf{3}} ; 1,1)$ & 3 & 0 & 0 & 0 & -1 & 0 & 0 & 0 & 1 & 0 & 0 & -3 & -1 & 0 & 1 & -1 & 0 \\
\hline \multirow[t]{4}{*}{$13^{\prime} T$} & $\mid(\overline{\mathbf{3}}, 1,1,1 ; 1,1)$ & 5 & -1 & 0 & 0 & 0 & 0 & 0 & 0 & 0 & 0 & -1 & -1 & 3 & -1 & 0 & 0 & 1 \\
\hline & $\mid(1, \overline{\mathbf{3}}, 1,1 ; 1,1)$ & 5 & 0 & -1 & 0 & 0 & 0 & 0 & 0 & 0 & -1 & 0 & -1 & 3 & 1 & 0 & 0 & -1 \\
\hline & $\|(1,1, \overline{\mathbf{3}}, 1 ; 1,1)$ & 5 & 0 & 0 & -1 & 0 & 0 & 0 & 0 & -1 & 0 & 0 & 3 & -1 & 0 & -1 & 1 & 0 \\
\hline & $\|(1,1,1, \overline{\mathbf{3}} ; 1,1)$ & 5 & 0 & 0 & 0 & -1 & 0 & 0 & -1 & 0 & 0 & 0 & 3 & -1 & 0 & 1 & -1 & 0 \\
\hline \multirow[t]{8}{*}{$23 U$} & $\mid(1,1,1,1 ; \mathbf{2}, 1)$ & 2 & 0 & 0 & 0 & 0 & 1 & 0 & 1 & 0 & 0 & 0 & -3 & 0 & -3 & 0 & -3 & 0 \\
\hline & $\mid(1,1,1,1 ; \mathbf{2}, 1)$ & 2 & 0 & 0 & 0 & 0 & 1 & 0 & -1 & 0 & 0 & 0 & 3 & 0 & -3 & 0 & -5 & 0 \\
\hline & $\|(1,1,1,1 ; \overline{\mathbf{2}}, 1)$ & 2 & 0 & 0 & 0 & 0 & -1 & 0 & 0 & 1 & 0 & 0 & -3 & 0 & 3 & 0 & 3 & 0 \\
\hline & $\mid(1,1,1,1 ; \overline{\mathbf{2}}, 1)$ & 2 & 0 & 0 & 0 & 0 & -1 & 0 & 0 & -1 & 0 & 0 & 3 & 0 & 3 & 0 & 5 & 0 \\
\hline & $\mid(1,1,1,1 ; 1,2)$ & 2 & 0 & 0 & 0 & 0 & 0 & 1 & 0 & 0 & 1 & 0 & 0 & -3 & 0 & -3 & 0 & -3 \\
\hline & $\mid(1,1,1,1 ; 1,2)$ & 2 & 0 & 0 & 0 & 0 & 0 & 1 & 0 & 0 & -1 & 0 & 0 & 3 & 0 & -3 & 0 & -5 \\
\hline & $\mid(1,1,1,1 ; 1, \overline{\mathbf{2}})$ & 2 & 0 & 0 & 0 & 0 & 0 & -1 & 0 & 0 & 0 & 1 & 0 & -3 & 0 & 3 & 0 & 3 \\
\hline & $\mid(1,1,1,1 ; 1, \overline{\mathbf{2}})$ & 2 & 0 & 0 & 0 & 0 & 0 & -1 & 0 & 0 & 0 & -1 & 0 & 3 & 0 & 3 & 0 & 5 \\
\hline \multirow[t]{4}{*}{$23 T$} & $\mid(1,1,1,1 ; \mathbf{2}, 1)$ & 1 & 0 & 0 & 0 & 0 & 1 & 0 & 0 & 0 & -1 & 0 & 0 & 3 & -3 & 0 & -4 & -1 \\
\hline & $\mid(1,1,1,1 ; \overline{\mathbf{2}}, 1)$ & 1 & 0 & 0 & 0 & 0 & -1 & 0 & 0 & 0 & 0 & -1 & 0 & 3 & 3 & 0 & 4 & 1 \\
\hline & $\|(1,1,1,1 ; 1,2)$ & 1 & 0 & 0 & 0 & 0 & 0 & 1 & -1 & 0 & 0 & 0 & 3 & 0 & 0 & -3 & -1 & -4 \\
\hline & $\|(1,1,1,1 ; 1, \overline{\mathbf{2}})$ & 1 & 0 & 0 & 0 & 0 & 0 & -1 & 0 & -1 & 0 & 0 & 3 & 0 & 0 & 3 & 1 & 4 \\
\hline \multirow[t]{6}{*}{$33^{\prime} U$} & $\|(1,1,1,1 ; 1,1)$ & 16 & 0 & 0 & 0 & 0 & 0 & 0 & 1 & 1 & 0 & 0 & -6 & 0 & 0 & 0 & 0 & 0 \\
\hline & $(1,1,1,1 ; 1,1)$ & 16 & 0 & 0 & 0 & 0 & 0 & 0 & 0 & 0 & 1 & 1 & 0 & -6 & 0 & 0 & 0 & 0 \\
\hline & $\mid(1,1,1,1 ; 1,1)$ & 6 & 0 & 0 & 0 & 0 & 0 & 0 & -2 & 0 & 0 & 0 & 6 & 0 & 0 & 0 & -2 & 0 \\
\hline & $\|(1,1,1,1 ; 1,1)$ & 6 & 0 & 0 & 0 & 0 & 0 & 0 & 0 & -2 & 0 & 0 & 6 & 0 & 0 & 0 & 2 & 0 \\
\hline & $\|(1,1,1,1 ; 1,1)$ & 6 & 0 & 0 & 0 & 0 & 0 & 0 & 0 & 0 & -2 & 0 & 0 & 6 & 0 & 0 & 0 & -2 \\
\hline & $\|(1,1,1,1 ; 1,1)$ & 6 & 0 & 0 & 0 & 0 & 0 & 0 & 0 & 0 & 0 & -2 & 0 & 6 & 0 & 0 & 0 & 2 \\
\hline \multirow[t]{2}{*}{$33^{\prime} T$} & $\mid(1,1,1,1 ; 1,1)$ & 8 & 0 & 0 & 0 & 0 & 0 & 0 & -1 & 0 & 0 & -1 & 3 & 3 & 0 & 0 & -1 & 1 \\
\hline & $\|(1,1,1,1 ; 1,1)$ & 8 & 0 & 0 & 0 & 0 & 0 & 0 & 0 & -1 & -1 & 0 & 3 & 3 & 0 & 0 & 1 & -1 \\
\hline
\end{tabular}

Table 6: Chiral fermionic spectrum for $\mathbb{Z}_{2}$ with $\left(n_{1}, m_{1}\right)=(1,1),\left(n_{2}, m_{2}\right)=(1,0),\left(n_{3}, m_{3}\right)=(4,1)$ and lattice aaa. The resulting gauge group is $S U(3)^{4} \times S U(2)^{2} \times U(1)^{10}$.

\section{References}


[1] J. Polchinski, Phys. Rev. Lett. 75 (1995) 4724 hep-th/9510017.

[2] A. Sagnotti, ROM2F-87-25 Talk presented at the Cargese Summer Institute on NonPerturbative Methods in Field Theory, Cargese, France, Jul 16-30, $198 \%$.

[3] G. Pradisi and A. Sagnotti, Phys. Lett. B 216 (1989) 59.

[4] J. Govaerts, Phys. Lett. B 220 (1989) 77.

[5] P. Horava, Nucl. Phys. B 327 (1989) 461.

[6] M. Bianchi and A. Sagnotti, Phys. Lett. B 247 (1990) 517.

[7] M. Bianchi and A. Sagnotti, Nucl. Phys. B 361 (1991) 519.

[8] E. G. Gimon and J. Polchinski, Phys. Rev. D 54 (1996) 1667 [hep-th/9601038].

[9] A. Dabholkar and J. Park, Nucl. Phys. B472 (1996) 207 hep-th/9602030].

[10] E. G. Gimon and C. V. Johnson, Nucl. Phys. B477 (1996) 715 hep-th/9604129].

[11] M. Berkooz and R. G. Leigh, Nucl. Phys. B483 (1997) 187 hep-th/9605049.

[12] J. D. Blum and A. Zaffaroni, Phys. Lett. B387 (1996) 71 hep-th/9607019].

[13] Z. Kakushadze and G. Shiu, Phys. Rev. D56 (1997) 3686 hep-th/9705163.

[14] G. Zwart, Nucl. Phys. B526 (1998) 378 hep-th/9708040].

[15] S. Förste and D. Ghoshal, Nucl. Phys. B527 (1998) 95 hep-th/9711039].

[16] D. O'Driscoll, hep-th/9801114.

[17] G. Aldazabal, A. Font, L. E. Ibanez and G. Violero, Nucl. Phys. B536 (1998) 29 [hepth/9804026.

[18] M. Berkooz, M. R. Douglas and R. G. Leigh, Nucl. Phys. B480 (1996) 265 hep-th/9606139.

[19] R. Blumenhagen, L. Görlich and B. Körs, Nucl. Phys. B569 (2000) 209 hep-th/9908130.

[20] M. Cvetic, M. Plumacher and J. Wang, JHEP 0004 (2000) 004 hep-th/9911021.

[21] R. Blumenhagen, L. Görlich and B. Körs, JHEP 0001 (2000) 040 hep-th/9912204.

[22] G. Pradisi, Nucl. Phys. B 575 (2000) 134 hep-th/9912218.

[23] S. Förste, G. Honecker and R. Schreyer, Nucl. Phys. B 593 (2001) 127 hep-th/0008250.

[24] B. Feng, Y. He, A. Karch and A. Uranga, hep-th/0103177.

[25] L. E. Ibanez, F. Marchesano and R. Rabadan, hep-th/0105155.

[26] I. Antoniadis, N. Arkani-Hamed, S. Dimopoulos and G. Dvali, Phys. Lett. B 436 (1998) 257 hep-ph/9804398.

[27] T. Banks and L. Susskind, hep-th/9511194.

[28] A. Sen, Class. Quant. Grav. 17 (2000) 1251.

[29] A. Lerda and R. Russo, Int. J. Mod. Phys. A 15 (2000) 771 hep-th/9905006]. 
[30] M. R. Gaberdiel, Class. Quant. Grav. 17 (2000) 3483 hep-th/0005029.

[31] I. Antoniadis, E. Dudas and A. Sagnotti, Nucl. Phys. B 544 (1999) 469 hep-th/9807011.

[32] S. Sugimoto, Prog. Theor. Phys. 102 (1999) 685 hep-th/9905159].

[33] I. Antoniadis, E. Dudas and A. Sagnotti, Phys. Lett. B 464 (1999) 38 hep-th/9908023.

[34] C. Angelantonj, Nucl. Phys. B 566 (2000) 126 [hep-th/9908064].

[35] G. Aldazabal and A. M. Uranga, JHEP 9910 (1999) 024 hep-th/9908072.

[36] C. Angelantonj, I. Antoniadis, G. D'Appollonio, E. Dudas and A. Sagnotti, Nucl. Phys. B 572 (2000) 36 [hep-th/9911081].

[37] S. Mukhi, N. V. Suryanarayana and D. Tong, JHEP 0003 (2000) 015 hep-th/0001066.

[38] C. Angelantonj, R. Blumenhagen and M. R. Gaberdiel, Nucl. Phys. B 589 (2000) 545 [hepth/0006033.

[39] C. Angelantonj, I. Antoniadis, E. Dudas and A. Sagnotti, Phys. Lett. B 489 (2000) 223 [hepth/0007090.

[40] R. Rabadan and A. M. Uranga, JHEP 0101 (2001) 029 hep-th/0009135.

[41] R. Blumenhagen, L. Görlich, B. Körs and D. Lüst, JHEP 0010 (2000) 006 hep-th/0007024].

[42] R. Blumenhagen, L. Görlich, B. Körs and D. Lüst, Fortsch. Phys. 49 (2001) 591 hepth/0010198.

[43] G. Aldazabal, S. Franco, L. E. Ibanez, R. Rabadan and A. M. Uranga, JHEP 0102 (2001) 047 hep-ph/0011132.

[44] G. Aldazabal, S. Franco, L. E. Ibanez, R. Rabadan and A. M. Uranga, hep-th/0011073.

[45] I. V. Vancea, JHEP 0104 (2001) 020 hep-th/0011251.

[46] R. Blumenhagen, B. Körs and D. Lüst, JHEP 0102 (2001) 030 hep-th/0012156].

[47] W. Fischler and L. Susskind, Phys. Lett. B 173 (1986) 262.

[48] E. Dudas and J. Mourad, Phys. Lett. B 486 (2000) 172 [hep-th/0004165].

[49] R. Blumenhagen and A. Font, Nucl. Phys. B 599 (2001) 241 hep-th/0011269.

[50] L. Randall and R. Sundrum, Phys. Rev. Lett. 83 (1999) 3370 hep-ph/9905221.

[51] J. Polchinski and Y. Cai, Nucl. Phys. B 296 (1988) 91.

[52] Z. Lalak, S. Lavignac and H. P. Nilles, Nucl. Phys. B 559 (1999) 48 hep-th/9903160. 